\title{
Regenerated Optic Fibers in Goldfish Reestablish a Crude Sectoral Order in the Visual Pathway
}

\author{
ROBERT BERNHARDT AND STEPHEN S. EASTER, JR. \\ Department of Biology, The University of Michigan, Ann Arbor, Michigan 48109-1048
}

\begin{abstract}
The goldfish optic pathway is regenerated after an optic nerve crush. We have examined the axonal topography of the regenerated pathway by labeling, with horseradish peroxidase (HRP), axons originating from retinal sectors or annuli. The positions of the labeled axons in the cross section of the pathway were compared to the normal and related to the factors that may influence axonal pathfinding.

The positions of retinal axons in the cross section of the normal pathway are predictable from the retinal addresses of the ganglion cells described by the polar coordinates $r$ (the distance from the optic disc) and $\theta$ (the sectoral or clockface position). The two coordinates map orthogonally onto the cross section of the pathway; $r$ varies monotonically along one axis; $\theta$ varies along a perpendicular axis.

The normal r-order, present in the nonregenerated stump of the experimental nerve, was severely degraded and perhaps lost entirely in the regenerated optic nerve, tract, and brachia. Sectoral order was also lost as the axons passed the crush site, but it was reestablished, albeit crudely, in the regenerated tract and brachia where axons tended to occupy positions appropriate to their dorsal, ventral, nasal, and temporal retinal origins. The exit sequence of the regenerated axons from the stratum opticum into the tectal neuropil was normal: temporal first, nasal last.

These results suggest that the regenerating fibers followed some $\theta$ specific cue located in the nonaxonal environment. It seems likely that the original axons probably followed the same cue. In contrast, the absence of $r$ order suggests that there is no $r$-specific cue for the regenerates to follow. It seems likely that the original r-order was a consequence of nonspecific influences-the orderly spatiotemporal growth of the retina and the existence of a permissive region for axonal growth.
\end{abstract}

Key words: axonal outgrowth, axonal guidance, retinotectal system

The spatial order of axons in the optic pathway of fish has received recent attention spurred by an interest in the phenomenon of axonal guidance and its possible role in the formation of ordered projections. Work from several laboratories has led to a consensus that the normal order is based on retinal polar coordinates (Scholes, '79; Rusoff and Easter, '80; Easter et al., '81; Bunt, '82; Stuermer and Easter, '84b; Bernhardt and Easter, '86; Fraley and Sharma, '86; Springer and Mednick, ' $86 \mathrm{a}, \mathrm{b}$ ).If we define the retinal polar coordinates of a ganglion cell as $r$, the distance from the optic nerve head to the soma, and $\theta$, the sectoral or clockface position of the soma (relative to some ray such as the one connecting the optic nerve head to the ventral pole), then we find that the axons occupy positions in the cross section of the pathway such that the retinal polar coordinates map orthogonally to one another. For example, in that portion of the optic nerve near the retina, axons from the central retina occupy the dorsal part of the cross section, and axons of successively more peripheral origins occupy successively more ventral positions in the cross section. The sectoral origins are mapped continuously along the nasotemporal axis: ventronasal axons on the extreme nasal side, dorsonasal next, dorsotemporal next, and ventrotemporal on the extreme temporal side.

Accepted June 8, 1988.

(c) 1988 ALAN R. LISS, INC. 
TABLE 1. Experimental Pathways Labeled by HRP Applications to the

\begin{tabular}{|c|c|c|c|}
\hline \multicolumn{4}{|c|}{ Retina } \\
\hline Pathway & $\begin{array}{c}\text { Survival interval } \\
\text { (days) }\end{array}$ & Sector labeled & $\begin{array}{l}\text { Illustrated } \\
\text { in Figure }\end{array}$ \\
\hline $11 / 21-1 r$ & 54 & Dorsal & \\
\hline $11 / 20-2 \mathrm{r}$ & 54 & Dorsal & \\
\hline $11 / 20-1 \mathrm{r}$ & 66 & Dorsal & $4 B$ \\
\hline $7 / 7 \cdot 1 \mathrm{r}$ & 116 & Dorsal & \\
\hline $11 / 21-11$ & 54 & Ventral & \\
\hline $11 / 20-21$ & 54 & Ventral & $4 A$ \\
\hline $11 / 20-21$ & 66 & Ventral & \\
\hline $7 / 7-2 r^{2}$ & 116 & Ventral & \\
\hline $11 / 21-1 \mathrm{r}$ & 54 & Temporal & \\
\hline $6 / 21-1 r$ & 100 & Temporal & $6 \mathrm{C}, 7 \mathrm{~A}$ \\
\hline $6 / 27-1 r$ & 114 & Temporal & \\
\hline $7 / 11-1 r$ & 120 & Temporal & \\
\hline $7 / 18-1 \mathrm{r}$ & 127 & Temporal & $2 \mathrm{~A}, \mathrm{C}, \mathrm{E}, 5 \mathrm{~B}, 6 \mathrm{D}$ \\
\hline $7 / 25-1 \mathrm{r}$ & 465 & Temporal & \\
\hline $1 / 21-11$ & 54 & Nasal & \\
\hline $6 / 20-1 \mathrm{r}$ & 100 & Nasal & $6 \mathrm{~B}$ \\
\hline $6 / 27-2 r$ & 114 & Nasal & $5 \mathrm{D}, 6 \mathrm{~A}, 7 \mathrm{~B}$ \\
\hline $7 / 11-2 \mathrm{r}^{*}$ & 120 & Nasal & \\
\hline $7 / 18-2 \mathrm{r}$ & 127 & Nasal & 3 \\
\hline $7 / 25-2 \mathrm{r}$ & 465 & Nasal & \\
\hline
\end{tabular}

${ }^{1}$ Sectoral order was discernible in all experimental pathways, with one exception (asterisk)

${ }^{2}$ Pathway lost.

The question of whether regenerating axons reestablish the original order has been addressed in both fish and amphibians, and the brief answer is that they do, but not very accurately (Attardi and Sperry, '63; Horder, '74; Meyer, '80; Stuermer and Easter, '84a; Stuermer, '86). But none of these inquiries as based on our current detailed knowledge of the spatial order in the normal pathway, and none has asked the question separately of the two axes. That is the subject of this paper.

In the companion paper (Bernhardt et al., '88), we address a related issue-the importance of axon-axon interactions in the genesis of spatial order. Brief preliminary accounts of parts of both papers have appeared (Bernhardt et al., '86; Bernhardt and Easter, '87; Easter and Bernhardt, '87).

\section{MATERIALS AND METHODS Optic nerve crush}

Goldfish, 50-70-mm standard body length, were anesthetized by immersion in $0.2 \%$ tricaine methane sulfonate, and the right optic nerve was crushed repeatedly with fine watchmaker's forceps until a clear gap (approximately 2 $\mathrm{mm}$ from the eye) separated the proximal and distal stumps. The nerve sheath was left intact. In some animals the left nerve was untouched; in others it was crushed in the same way as the right and thus provided a second experimental pathway. After surgery, the fish were revived and returned to their tanks. The results are based on 34 experimental pathways from 26 fish, which survived for 54--465 days postcrush.

\section{Sectoral labeling}

After a variable survival period the fish were again anesthetized. In both eyes, the same retinal sector was labeled by inserting a pellet of Gelfoam (Upjohn) soaked with horseradish peroxidase (HRP) through a small incision in the sclera. This protocol labels a narrow sector $\left(5-10^{\circ}\right.$ subtense $)$ of ganglion cells extending from the lesion site to the retinal margin and their axons (Bernhardt and Easter, '86). Axons labeled in this way constitute a "$\theta$-group." The reti-

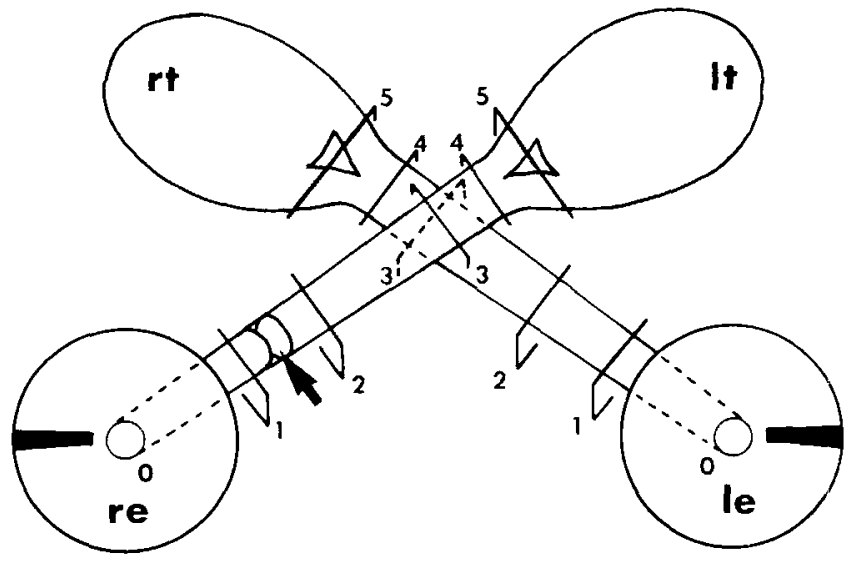

Fig. 1. Schematic of the fish retinotectal projection indicating the levels of the optic pathway illustrated in subsequent Figures: 0 , optic nerve head; 1 , distal optic nerve; 2 , proximal optic nerve; 3 , nerve-tract junction; 4 , tract; 5 , brachia of the tract. The arrow indicates the site of the optic nerve lesion. The black truncated wedge in both retinae indicates the approximate area of retinal ganglion cells labeled by a temporal sectoral application of HRP. Ie, left eye; re, right eye; lt, left tectum; rt, right tectum.

TABLE 2. Experimental Pathways Labeled by HRP Applications to the Optic Nerve

\begin{tabular}{lcl}
\hline Pathway & $\begin{array}{c}\text { Survival Interval } \\
\text { (days) }\end{array}$ & \multicolumn{1}{c}{$\begin{array}{c}\text { Illustrated } \\
\text { in Figure }\end{array}$} \\
\hline $9 / 22-1 \mathrm{r}$ & 54 & \\
$9 / 22-11$ & 54 & $8 \mathrm{~A}, 13 \mathrm{~A}$ \\
$9 / 15-1 \mathrm{r}$ & 60 & $9 \mathrm{C}, 11 \mathrm{~A}$ \\
$9 / 15-1 \mathrm{l}$ & 60 & \\
$9 / 28-1 \mathrm{r}$ & 60 & $9 \mathrm{~A}$ \\
$9 / 28-11$ & 60 & $12 \mathrm{~A}, \mathrm{~B}$ \\
$4 / 18-1 \mathrm{r}$ & 65 & \\
$4 / 18-1 \mathrm{l}$ & 65 & $12 \mathrm{C}$ \\
$4 / 18-2 \mathrm{r}$ & 65 & \\
$4 / 18-2 \mathrm{l}$ & 65 & $9 \mathrm{~B}$ \\
$4 / 25-1 \mathrm{r}$ & 196 & \\
$4 / 25-1 \mathrm{l}$ & 196 & $9 \mathrm{D}, 12 \mathrm{D}$ \\
$4 / 25-2 \mathrm{r}$ & 196 & \\
$4 / 25-2 \mathrm{l}$ & 196 & \\
\hline
\end{tabular}

${ }^{1}$ Normal r-order was absent in all experimental pathways.

nal origins of the labeled axons in the regenerated portion of the pathway are therefore known, and their locations in the cross section of a regenerated pathway can be compared with axons of similar origins in the intact pathway. A list of the experimental pathways is given in Table 1 .

\section{Annular labeling}

In anesthetized animals the eye was deflected and the optic nerve was exposed in the orbit. A pin coated with HRP was inserted partway into the nerve on the retinal side of the crush. This procedure backfills somata occupying one or more partial annuli of varying angular extent in the corresponding retina (Rusoff and Easter, '80; Bernhardt and Easter, '86; Fraley and Sharma, '86) and their axons. Axons labeled in this way constitute an "r-group," and their locations in the regenerated pathway can be compared to axons of similar origins in the intact pathway. A list of the experimental pathways is given in Table 2. 

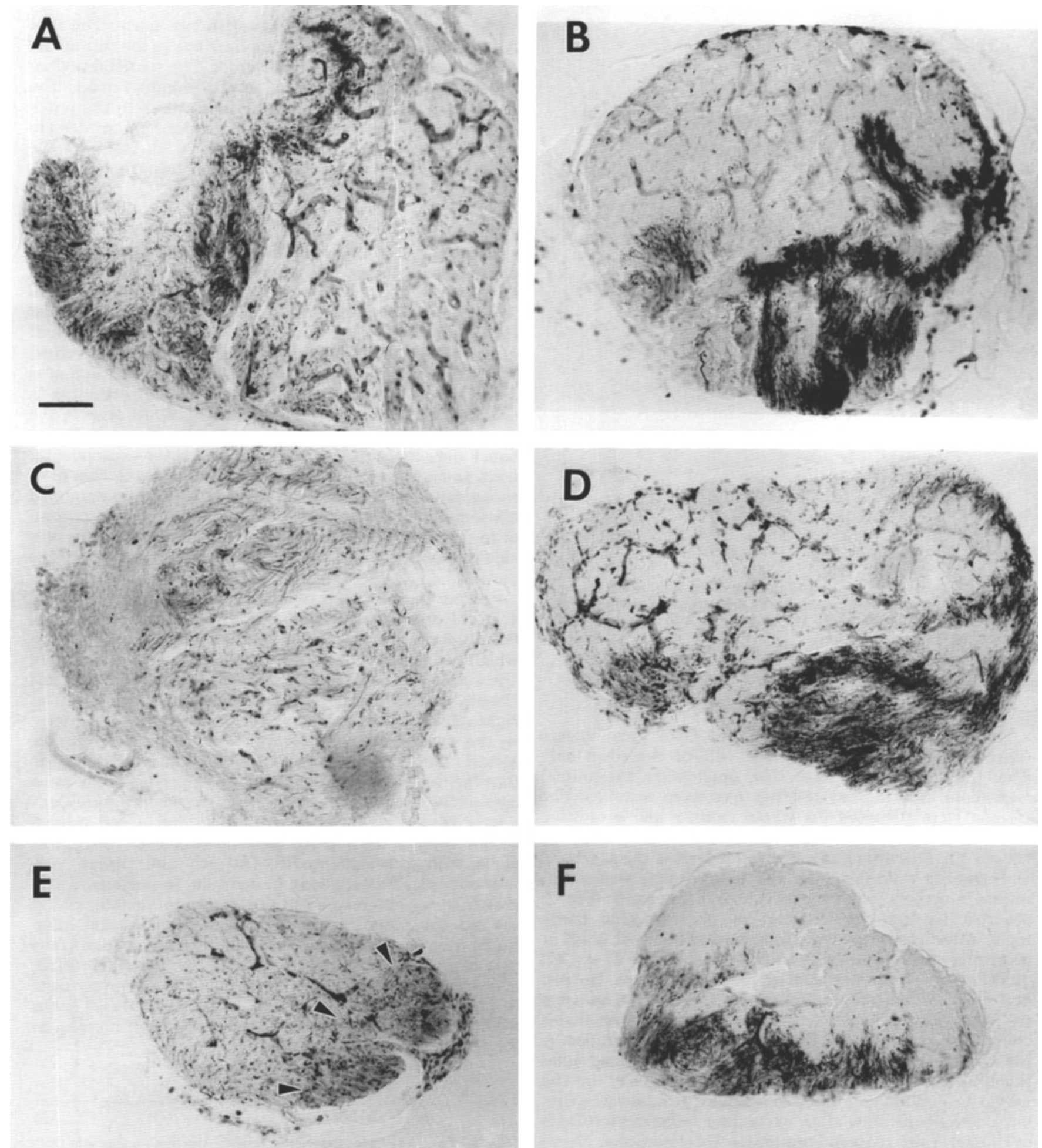

Fig. 2. A comparison of regenerated (127 days postcrush) and intact temporal $\theta$-groups at levels $1-3$. Micrographs of cross sections through the experimental (A,C,E) and control (B,D,F) pathways of one animal. Orientation of the sections A-D is dorsal up, nasal toward the center. In $\mathbf{E}$ and $\mathbf{F}$ rostrodorsal is up, medial away from the center. Calibration bar for all panels is $100 \mu \mathrm{m}$. A: In the distal (nonregenerated) portion of the experimental optic nerve (level 1), the $\theta$-group forms a dense band, occupying roughly the same position as a corresponding $\theta$-group in the control nerve (B). B:

Distal control nerve (level 1). C: In the proximal (regenerated) portion of the experimental nerve (level 2), the labeled axons are scattered throughout the cross section. D: Proximal control nerve (level 2) The labeled axons are clustered. E: Nerve-tract junction (level 3). The experimental $\theta$-group shows some reaggregation (arrowheads), but additional axons are scattered outside the main population. F: Nerve-tract junction (level 3). The intact $\theta$ group is clustered. There are practically no axons outside the main group. 


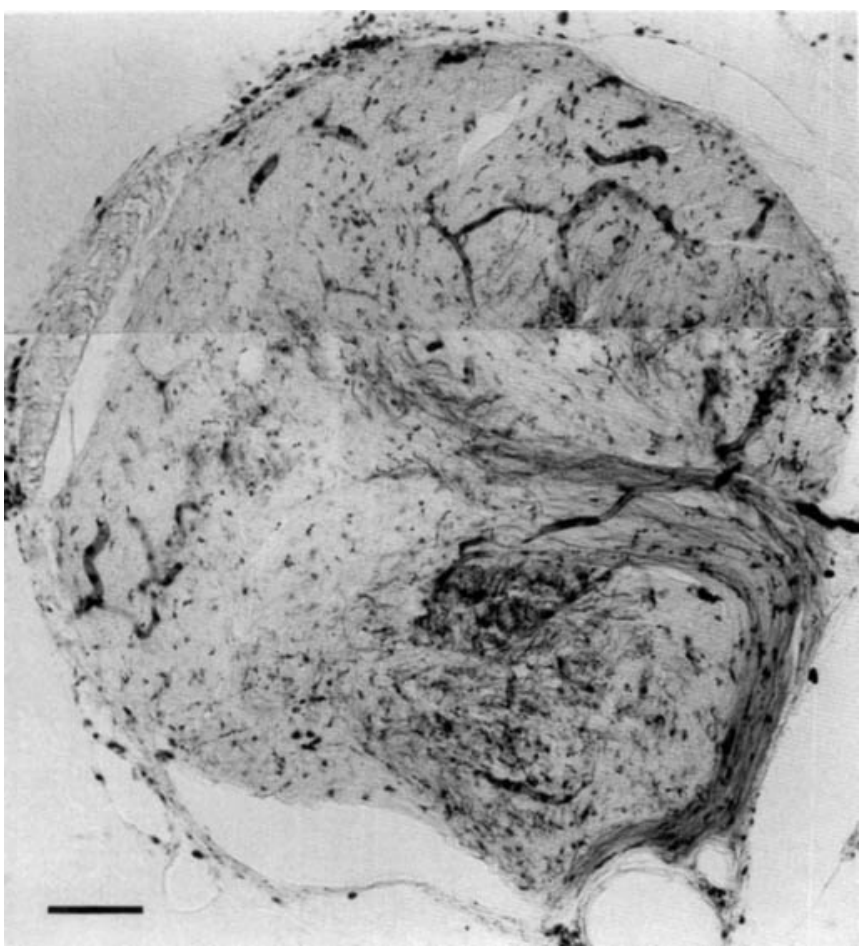

Fig. 3. The dispersion of axons at the crush site. A cross section at the crush site in an experimental nerve (127 days postcrush). A temporal $\theta$ group was labeled intraretinally. Dorsal is up, nasal to the right. Calibration bar is $100 \mu \mathrm{m}$

\section{HRP histochemistry}

The detailed reaction protocol has been given before (Bernhardt and Easter, '86) and will be described only briefly. Two or 3 days after HRP application, the animal was again anesthetized and the eyes were removed. The retinae were prepared for whole mounts and processed, with diaminobenzidine (DAB) (Adams, '81). The brain with the optic nerves attached was dissected out of the skull and embedded in gelatin/sucrose. Sections $(40 \mu \mathrm{m})$ were cut at an angle perpendicular to the course of the axons (Fig. 1; see also Bernhardt and Easter, '86) on a freezing microtome. Alternate series were processed either with $\mathrm{DAB}$ or according to the Hanker-Yates protocol (Hanker et al., '77) and slightly counterstained with methylene green. The two protocols yielded comparable results but differed in some respects. The DAB reaction stained axons crisply but also stained the blood vessels. Blood vessels were not stained by the Hanker-Yates reaction, which, however, stained additional cells, probably macrophages, particularly in the nerve. In our hands other chromogens (o-dianisidine and tetramethylbenzidine) stain axons less reliably than DAB or Hanker-Yates reagent (unpublished observations). Nerve sections are illustrated by DAB material. The more heavily vascularized brachia of the optic tract are illustrated by Hanker-Yates material. Weakly labeled, scattered axons were sometimes difficult to identify on photomicrographs, even though they were clearly seen in the microscope when focusing through the section at high magnification. Therefore, some sections are illustrated by camera lucida drawings.

\section{RESULTS}

The observations are presented in two parts. The first describes the results of HRP applications to the retina and evaluates the regeneration of $\theta$-order. The second describes the applications to the nerve and evaluates r-order. The description proceeds from the distal (retinal) to the proximal (tectal) end of the pathway with specific references to the levels, 0-5, illustrated in Figure 1. To facilitate the interpretation of the regenerated axon topography, exam. ples of normal order are included.

\section{$\theta$-order}

Axons of $\operatorname{common} \theta$ were clustered at level 1, in the distal, nonregenerated part of the experimental pathway and in the control pathway (Fig. 2A,B). More proximally, at level 2 , the labeled regenerated axons were widely dispersed throughout the cross section (Fig. 2C), quite different from normal (Fig. 2D). The dispersion originated at the crush site, where axons of common $\theta$ became entangled in a neuroma. Labeled axons formed large swirls, many running in the plane of the transverse section (Fig. 3). At level 3, the regenerated axons were rearranged; labeled axons of common $\theta$ were concentrated in a subregion of the experimental tract as on the normal side (Fig. 2E,F). Despite this rearrangement, the dispersion of axons in the $\theta$-group remained abnormally large in the experimental pathway. Inspection at higher magnification revealed many labeled axons outside the main group; very few such axons were seen in the normal.

Further evidence of a rearrangement was found at level 4 . In the normal pathway this is where axons change positions to form a new $\theta$-map, different from that in the nerve, which anticipates the tectal innervation pattern (Scholes, '79; Springer and Mednick, ' $86 \mathrm{~b}$; see also Bernhardt and Easter, '86). In the normal pathway, many axons ran at right angles to one another to form a herringbone pattern. In the experimental pathway a similar pattern was observed. This particular pattern in this location suggests that the regenerated axons probably responded to the same cues as the normal ones had done during their outgrowth.

Most of the regenerated axons from dorsal and ventral retina occupied the appropriate brachium (Fig. 4), in agreement with previous studies (Attardi and Sperry, '63; Stuermer and Easter, '84a). In addition, the regenerated $\theta$ groups were concentrated in the appropriate subregions of the brachial cross sections (Fig. 5). Temporal axons (Fig. $5 \mathrm{~A}, \mathrm{~B})$ were clustered near nucleus pretectalis superficialis pars magnocellularis (nppm), which separates the brachia, while nasal axons (Fig. 5C,D) were on the side away from it. The regenerated brachia had many more labeled axons outside the cluster than were seen on the normal side, so the reordering was only crude.

Fig. 4. Two regenerated $\theta$-groups at level 5. A pair of micrographs $(A, B)$ of cross sections through two pairs of experimental brachia. Rostrodorsal is up, medial to the left; db, dorsal brachium; vb, ventral brachium; nppm, nucleus pretectalis superficialis pars magnocellularis; $t l$, torus longitudinalis; asterisks, nucleus pretectalis superficialis pars parvicellularis (nppp). Calibration bar is $100 \mu \mathrm{m}$. A: A ventral $\theta$-group (54 days postcrush) is concentrated in the (appropriate) dorsal brachium (db), but some of the regenerated axons are in the (inappropriate) ventral brachium (vb). B: A dorsal $\theta$-group ( 66 days postcrush) is concentrated in the (appropriate) ventral brachium (vb). A few labeled axons are also found in the (inappropriate) dorsal brachium (db) but are difficult to distinguish in the micrograph. 

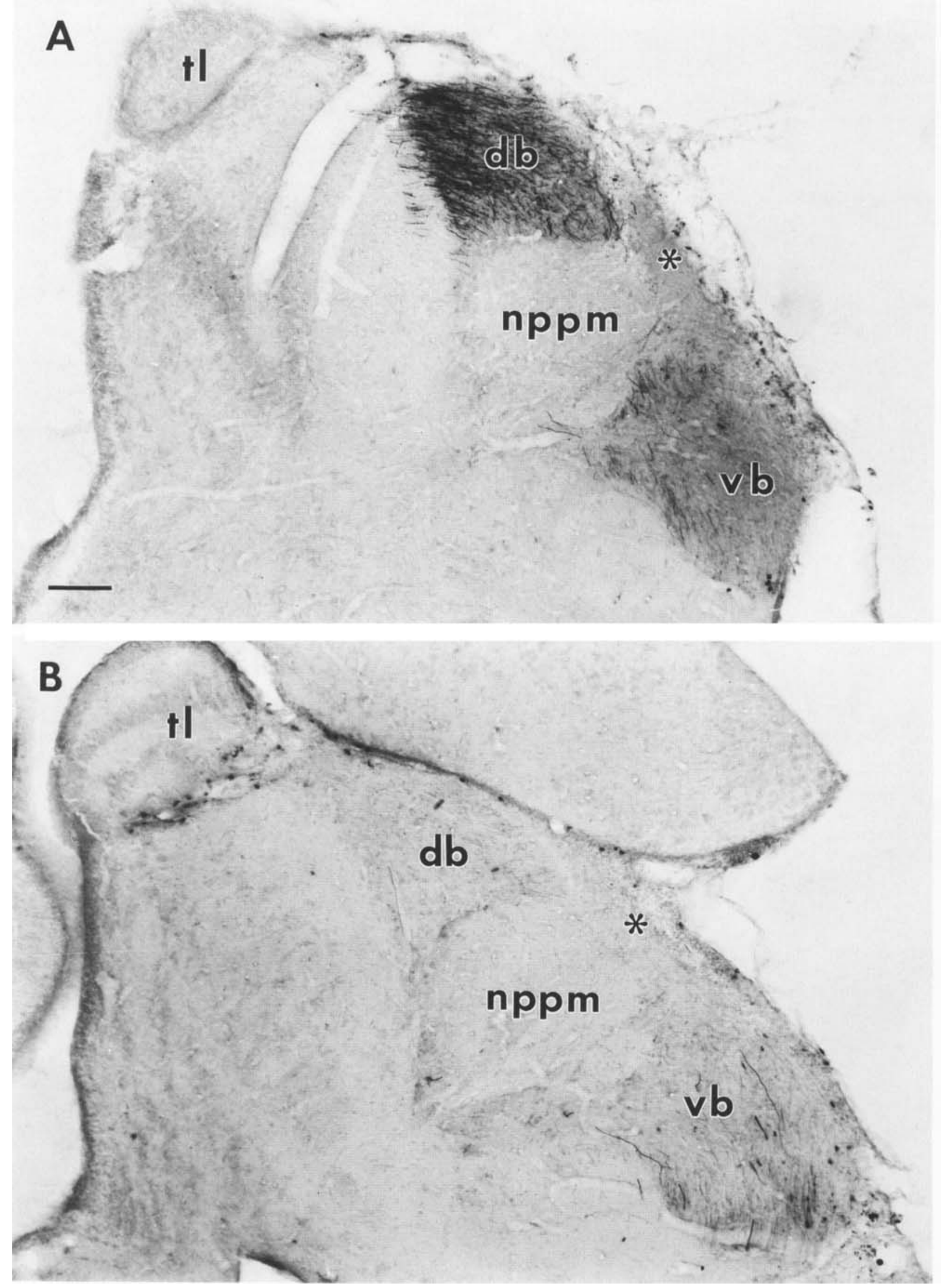


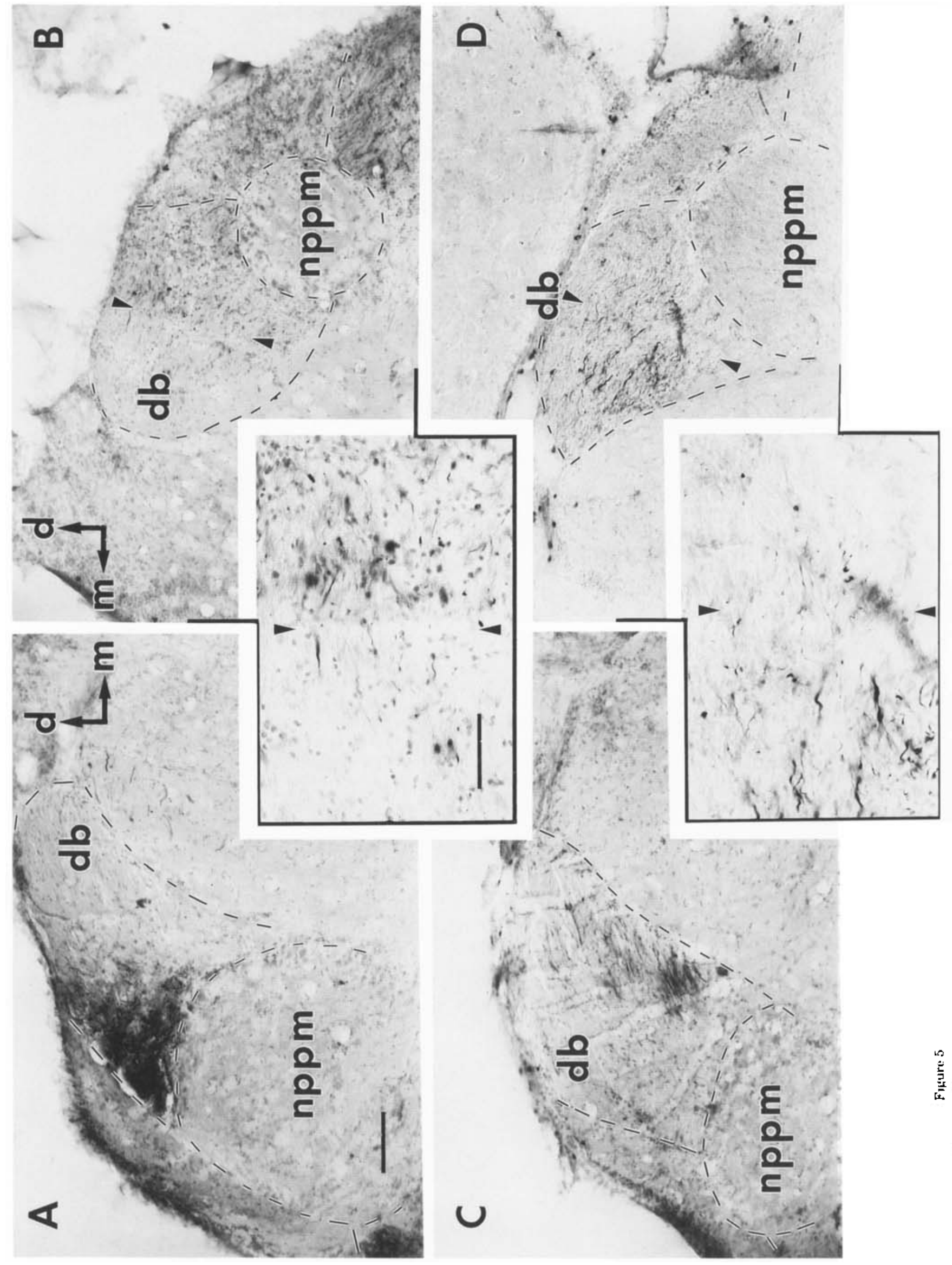



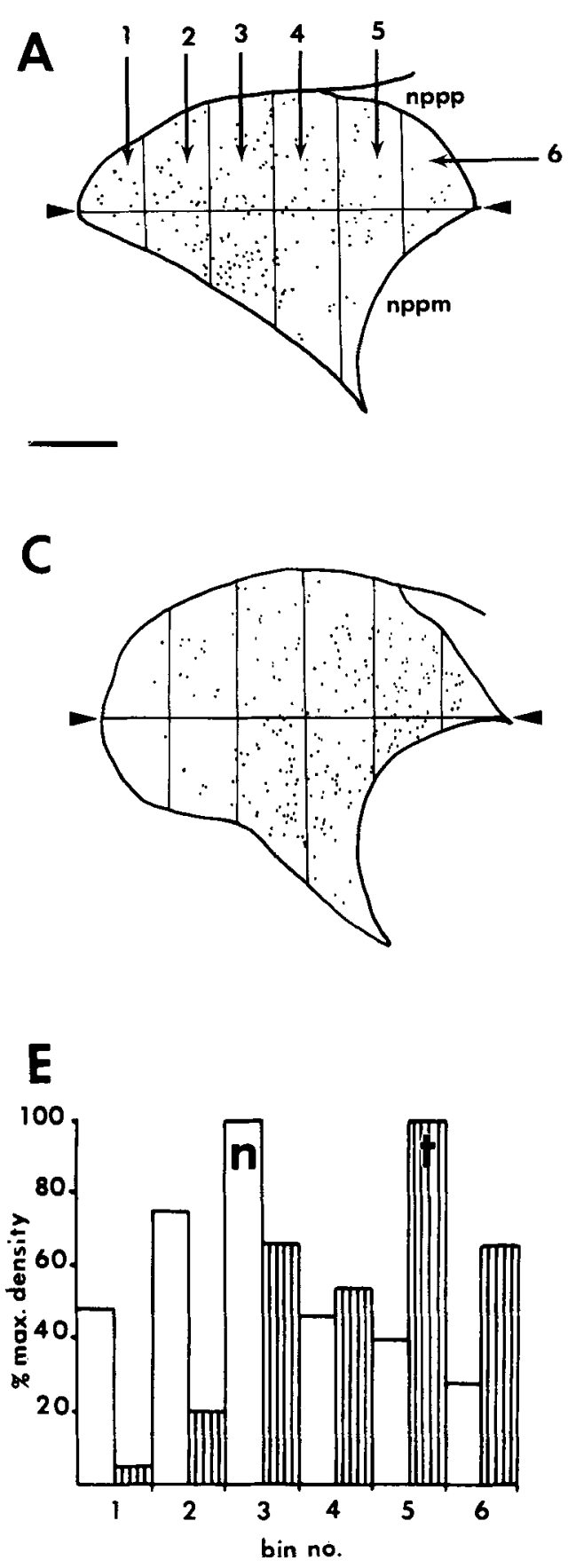

Fig. 5. A comparison of regenerated and intact $\theta$-groups at level 5 . Micrographs illustrating cross sections through two intact $(A, C)$ and two experimental (B,D) dorsal brachia. Rostrodorsal (d) is up, medial (m) to the center. The contours of the brachia are outlined. Compare to Figure 4. Calibration bar is $100 \mu \mathrm{m}$ for main panels, $\mathbf{5 0} \mu \mathrm{m}$ for insets. A: Section through a right, intact brachium, same animal as in Figure 2. The temporal $\theta$-group is clustered near nppm. B: Section through the left, experimental brachium (127 days post crush), same animal as in A. Although axons of the temporal $\theta$-group are more scattered than in the control (A), they are concentrated to the right of the arrowheads near nppm. The inhomogeneity is apparent in the inset-a more magnified view of the region between the arrowheads. $C$ : Section through a right, intact brachium. The nasal $\theta$-group is clustered far from nppm. D: Section through a left, experimental brachium (different animal from $\mathrm{C}$ ). The nasal $\theta$-group (114 days post crush) is more scattered than the control $(\mathrm{C})$, but axons are concentrated in the appropriate subregion of the brachium (to the left of the arrowheads). This clustering is illustrated in the inset-a more magnified view of the region between the arrowheads.
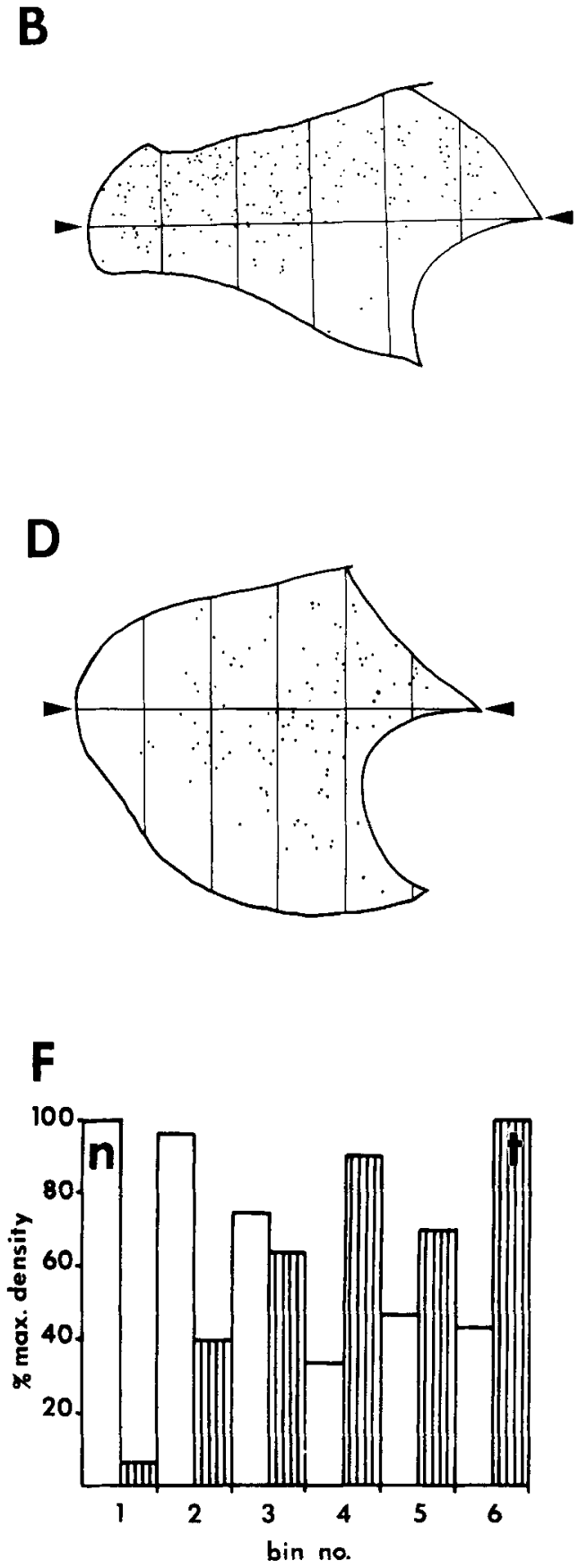

Fig. 6. Quantitative evidence for the reestablishment of sectoral order at level 5. A-D are camera lucida tracings of four experimental dorsal brachia with individual labeled axons indicated as dots. The bar graphs (E,F) plot the densities of these axons as a function of position in the cross section. Calibration bar for panels A-D is $100 \mu \mathrm{m}$. A,B: Axons from the nasal retinal sector, 114 (A) and 104 days postcrush (B). A micrograph of the brachium traced in $A$ is shown in Figure 5D. C,D: Axons from the temporal retinal sector, $100(\mathrm{C})$ and 127 days postcrush (D). A micrograph of the brachium traced in $\mathrm{D}$ is shown in Figure $5 \mathrm{~B}$. The same animal is also illustrated in Figure 2. E,F: Densities of labeled axons were determined in six bins of equal width (1-6), constructed perpendicular to the long axis of the brachium (defined to extend from the intersection of nppm and nppp to the farthest point on the opposite side of the brachium; compare A, long axis indicated by arrowheads). These bins are roughly congruent with normal $\theta$ groups (see Fig. 5A,C). The bar graphs plot the densities (axons per unit area expressed as the percentage of maximum density in the brachium) within each bin. $\mathrm{E}$ is a comparison of the nasal and temporal axons illustrated in $\mathrm{A}$ and $\mathrm{C}$. F is a comparison of the nasal and temporal axons illustrated in B and D. The peaks of the distributions of nasal (n) and temporal (t) axons are separated, and each population is concentrated in the appropriate subregion of the brachium. 


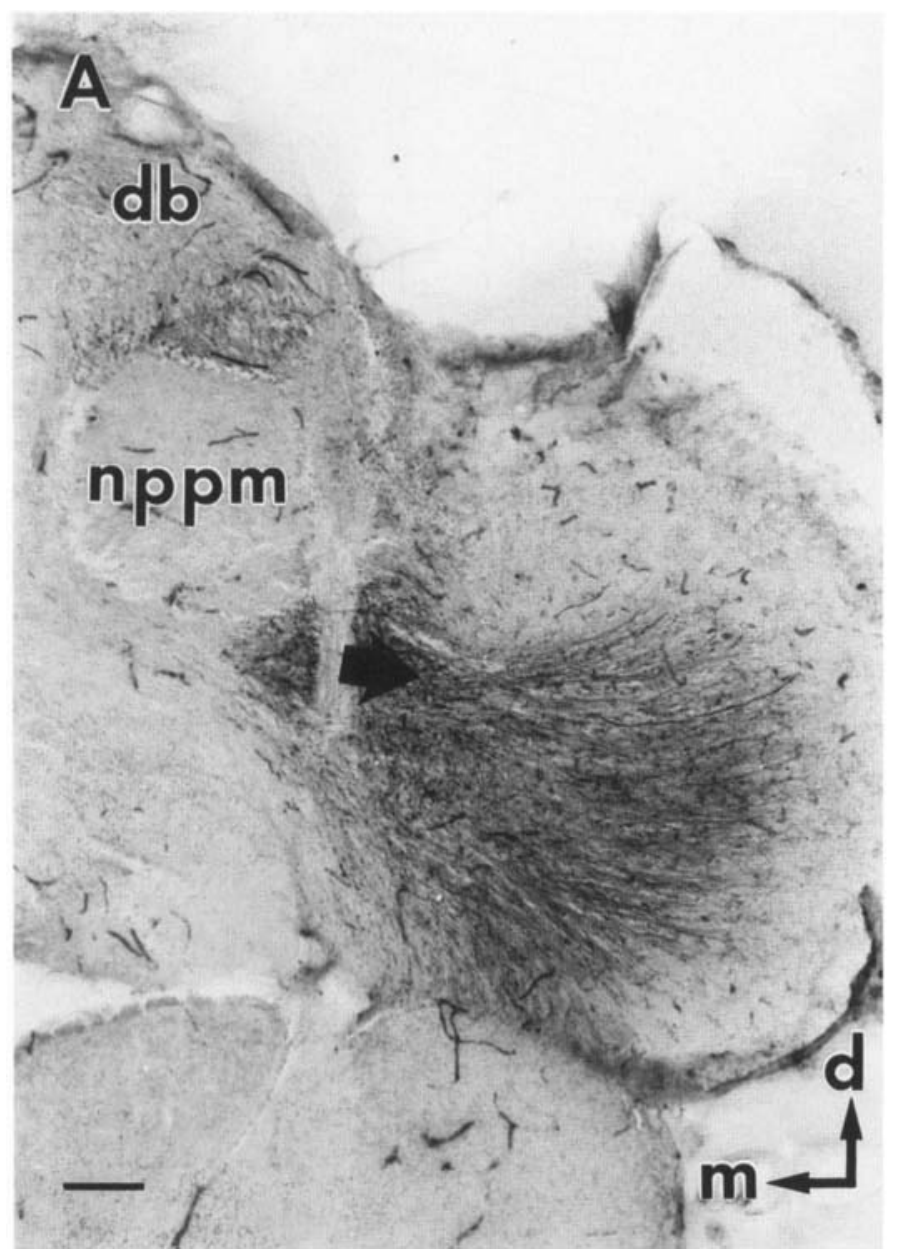

C

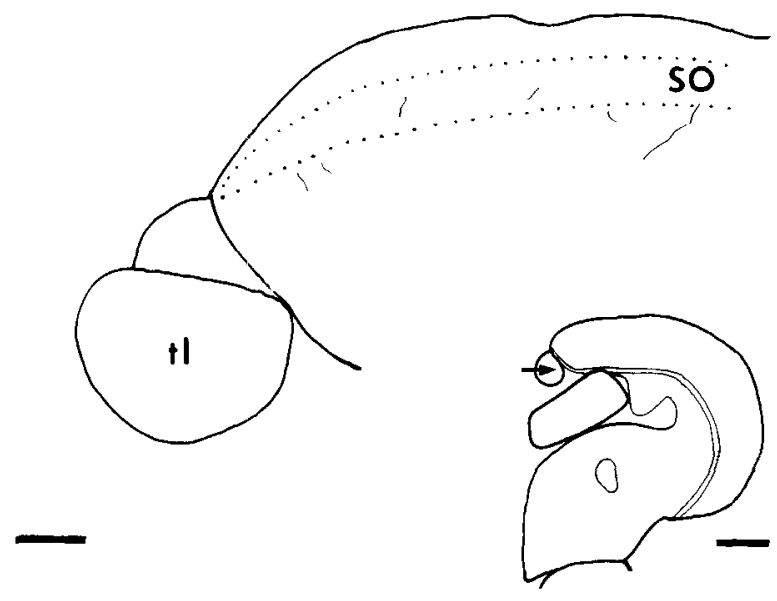

Fig. 7. Roughly normal tectal innervation patterns by regenerated $\theta$ groups. Micrographs (A,B) and camera lucida drawings $(C, D)$ of cross sections through two (left) tecta, illustrating the innervation pattern established by regenerated temporal $(A, C)$ and nasal axons $(B, D)$, at two levels, one rostral $(A, B)$ and the other more caudal $(C, D)$. $A$ and $C$ are from the same animal as Figure 6C. B and D are from the same animal as Figures $5 \mathrm{D}$ and $6 \mathrm{~A}$. Rostrodorsal (d) is up, medial $(\mathrm{m})$ to the left. A: At the rostral tectal pole temporal axons ( 100 days postcrush) have exited from the ventral brachium into the tectum (arrow). The dorsal brachium (db) has not yet fused with the tectum, and its labeled axons are clustered near nppm.

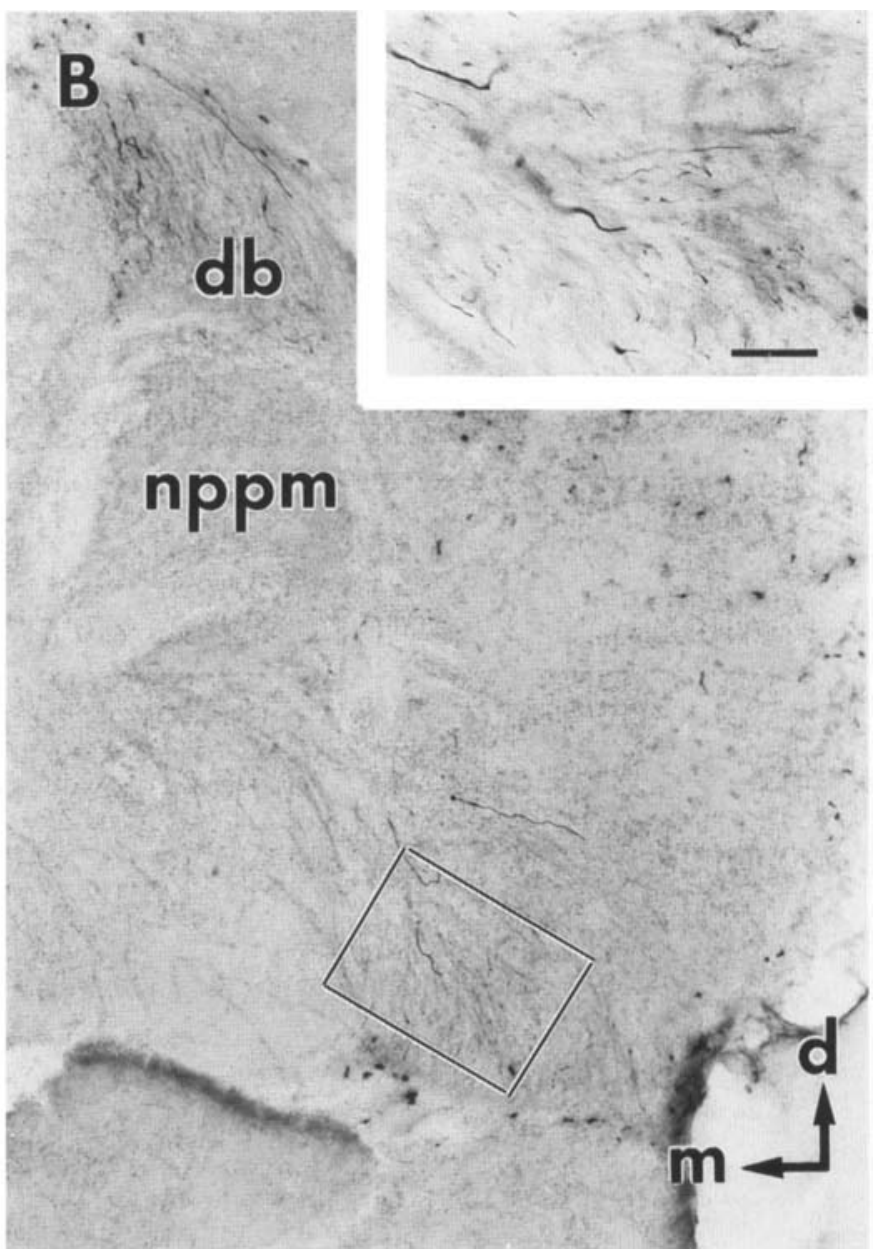

D

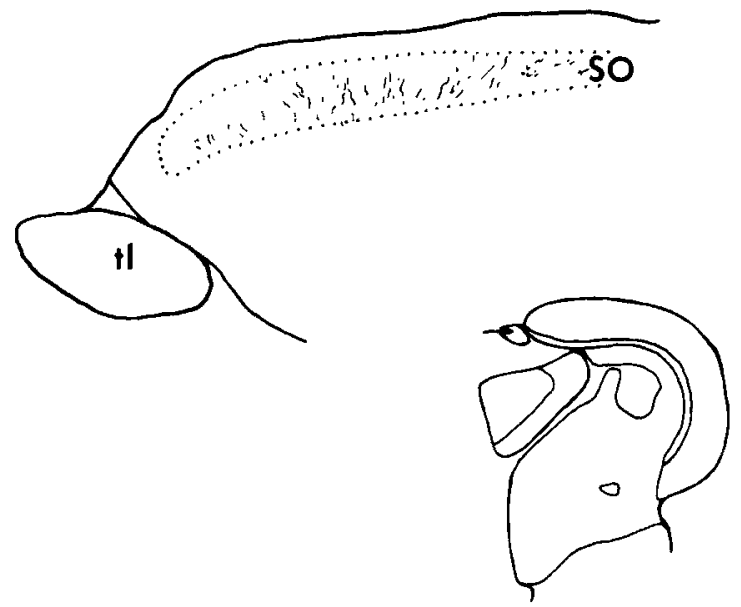

Calibration bar is $100 \mu \mathrm{m}$. B: Nasal axons in the ventral brachium (114 days postcrush) do not innervate the rostral tectal pole but remain at the ventrolateral edge of the tectum (box and inset). Same magnification as in A. Calibration bar in inset is $50 \mu \mathrm{m}$. C: Halfway along the rostrocaudal extent of the tectum very few temporal axons are still found in the tectal stratum opticum (SO). Calibration bar is $100 \mu \mathrm{m}$. The inset shows a low power overview of the same section. $t$, torus longitudinalis (also indicated by arrow in inset). Calibration bar in inset is $500 \mu \mathrm{m}$. D: At a level corresponding to $\mathrm{C}$, many nasal axons occupy the fiber layer (SO). Same conventions as in $\mathrm{C}$. 
This intrabrachial reordering was scored "blindly" in 12 experimental pathways, at 54-456 days past crush (see Table 1). An observer (S.S.E.) familiar with the normal order but ignorant of the particular brains that he scored attempted to identify the retinal labeling site as either nasal or temporal. He was correct in 11 and incorrect in only one, thus providing further support for $\theta$-order.

The visual impression of reordering was confirmed quantitatively. We drew individual labeled axons in the dorsal brachia of four experimental animals at 100-127 days postcrush (Fig. 6A-D). (Normal brachia could not be drawn similarly because the labeled axons were so tightly clustered and so numerous that they could not be reliably discerned individually.) The densities of labeled axons are plotted, as a function of position in the cross sections, in Figure $6 \mathrm{E}, \mathrm{F}$. These graphs reveal a sizable population of ectopic axons, but they show a clear trend toward the reestablishment of normal order (i.e., that shown in Fig. 5A and $\mathbf{C}$ ).

The innervation of the tectum by regenerated axons was also ordered as in the normal, albeit crudely. In the normal fish, temporal axons from peripheral retina leave the brachium and stratum opticum rostrally and innervate rostral tectum, in contrast to peripheral nasal axons, which remain in the stratum opticum until they reach caudal tectum, where they exit and terminate (Stuermer and Easter, ' $84 \mathrm{~b}$ ). The same pattern was noted in the regenerate. Temporal axons exited the brachium rostrally (Fig. 7A) and were absent more caudally (Fig. 7C). Nasal axons did not exit rostrally but remained in the brachia (Fig. 7B) and were seen more caudally in the stratum opticum (Fig. 7D).

In summary, the path of the regenerated $\theta$-group of axons is crudely similar to the normal. Although the group disperses in the nerve, downstream from the lesion (Figs. 2, 3 ), it reaggregates at the nerve/tract junction (Fig. 2), rearranges just outside the brain, occupies the appropriate subregion of the appropriate brachium (Figs. 4-6), and exits the pathway in the appropriate part of the tectum (Fig. 7). We emphasize that the number of "misplaced" axons (those outside the main group) is much greater than normal. But the clear conclusion is that regenerating axons tend to occupy the same subregions of the optic pathway, with respect to the $\theta$-variable, as their intact predecessors did in the normal fish.

\section{r-order}

To what extent is order reestablished along the r-axis? The regenerated r-groups are documented in Figures 8-12, a set of photomicrographs, drawings, and histograms from experimental pathways sampled at 54-196 days postcrush at the same levels as the $\theta$-groups and compared to the normal pattern.

Axons of common $r$ were tightly clustered on the retinal side of the lesion and dispersed in the regenerated nerve. The regenerated r-group often occupied an abnormal position in the cross section of the pathway. The distribution of labeled axons originating from almost complete retinal annuli (Fig. 8A,B) are illustrated for both an experimental and an intact pathway in Figure $8 \mathrm{C}-\mathrm{H}$. Near the nerve head (level 0 ) both the experimental (Fig. 8C) and the normal groups of axons (Fig. 8D) extended nasotemporally. Over the remainder of the nerve the absence of absolute landmarks precludes a direct comparison. But at level 3 (Fig. 8E,F) the distinct geometry of the cross section allows such a comparison, and the location and orientation of the experimental group is clearly not normal. The normal group of Figure $8 \mathrm{~F}$ is orthogonal to the regenerated group of Figure $8 \mathrm{E}$. More proximally in the tract (level 4) the alignment of the regenerated r-groups varied. Some extended roughly parallel to the pial surface, much like the normal (Fig. $8 \mathrm{H}$ ), or were concentrated in the correct region without being properly aligned (Fig. 9A), while most others (Fig. 9B-D) were wholly abnormal. We conclude that r-groups tend not to regenerate in the same part of the cross section of the pathway that they previously occupied.

In the brachia (level 5) the r-groups were strikingly different in the experimental and normal animals. The normal axons formed a band in each brachium that extended par. allel to the pial surface, confined along the superficialdeep axis of the brachia (Fig. 10B; see also Bernhardt and Easter, '86). The normal $r$-order is precise and allows the resolution of two well-separated r-bands in the brachia if two separate annuli have been labeled in the retina (Fig. 11B). The regenerated axons did not form comparable $r$ bands. The labeled axons were scattered all along the superficial-deep axis of the brachia (Figs, 10A, 11A). We attempted to evaluate this impression of intrabrachial disorder "blindly," as we had done for $\theta$-order, in sections at the level of the nppm in seven experimental pathways. The observer (S.S.E.) was familiar with the normal map of retinal partial annuli into the brachial cross section but was unaware of the locations of the labeled partial annuli in these seven cases. In most cases, he was unable to assign the radius of the labeled annulus more accurately than within the inner or outer $80 \%$ of the retina. Even with such imprecise limits, two of the guesses were incorrect. The difficulty of making the judgments, and the poor results, support the visual impressions described above.

We attempted to evaluate the intrabrachial disorder objectively, as we had done for $\theta$-order in Figure 6. Most of the r-groups were too heavily labeled to count individual fibers, but in three lightly labeled pathways we could do so, and the results are illustrated in Figure 12. The histograms show broad peaks, indicating that the regenerated axons that were close neighbors in the nonregenerated portion of the pathway were not randomly dispersed throughout the brachium. However, despite the evidence for some weak cohesion among the axons, the histograms revealed no consistent relationship between the annular origin of the axons and their positions along the superficial-deep axis of the brachium. Compare, for instance, the location of the outer band of labeled axons in Figure 11B with the broad distribution of labeled axons in Figure 12A and B (at 65 days postcrush). Both sets of axons arose from very similar retinal locations. Likewise, the two very different distributions of Figure 12C (at 65 days postcrush) and D (at 196 days postcrush) arose from similar retinal regions. This confirms the impressions from the "blind" scoring. It contrasts with our findings for $\theta$, which revealed a consistent, predictable relationship between sectoral origins of axons and their positions in the cross-sectioned brachia.

In the tectal fiber layer the regenerated axons of common $r$ were evenly scattered (Fig. $13 \mathrm{~A}$ ) in contrast to the normal, in which they were clustered in a few distinct bundles (Fig. 13B).

In summary $\theta$-groups dispersed in the nerve but then consistently tended to reacquire normal locations along the $\theta$-axis in the tract, brachium, and tectum; r-groups dispersed in the nerve, continued to do so in the tract, brachia, and tectal fiber layer, and showed no consistent tendency to coalesce into a normal pattern. 

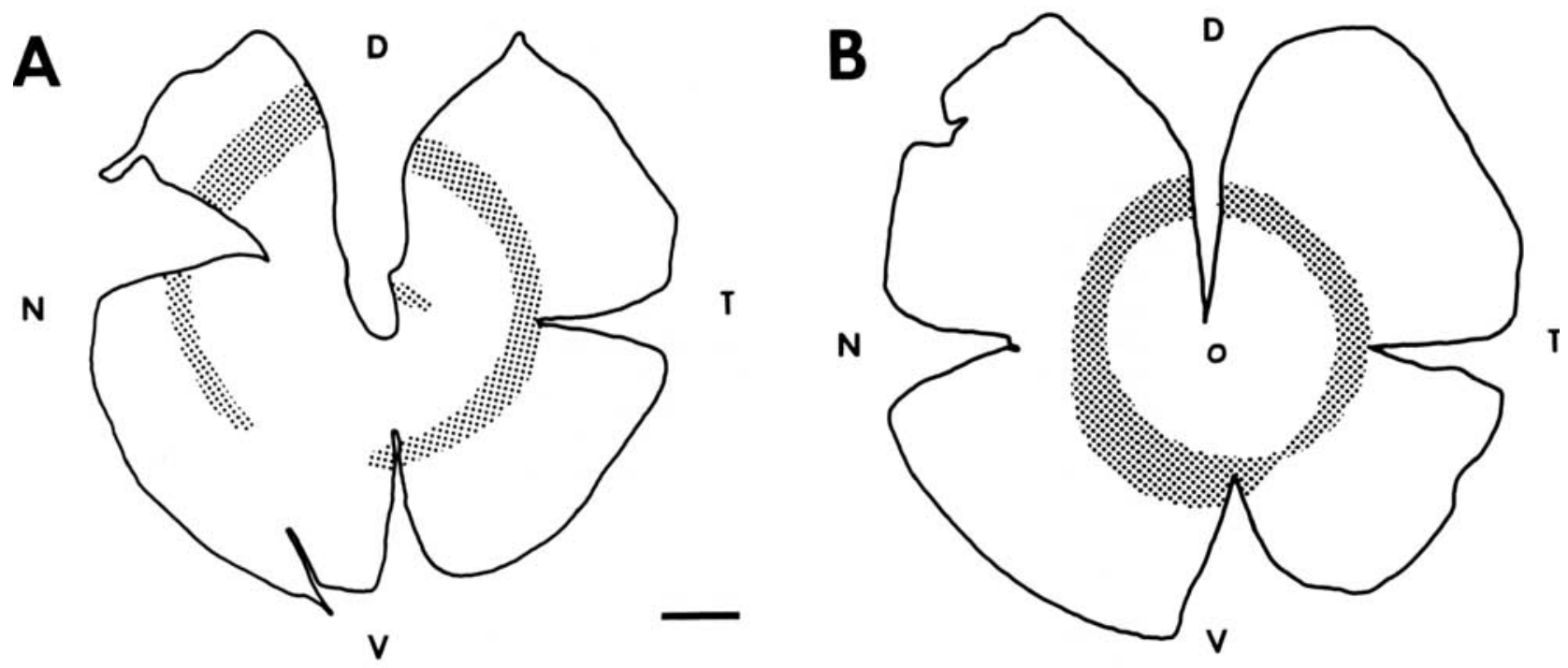

C

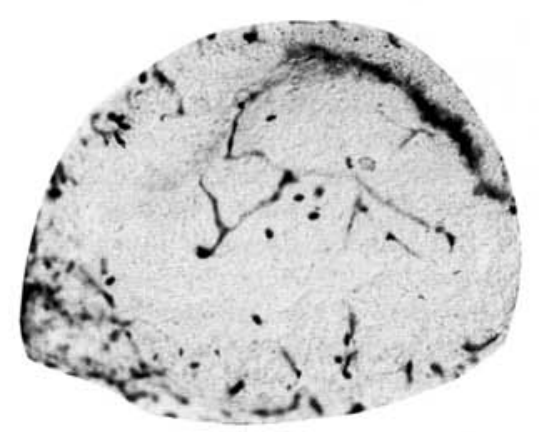

D

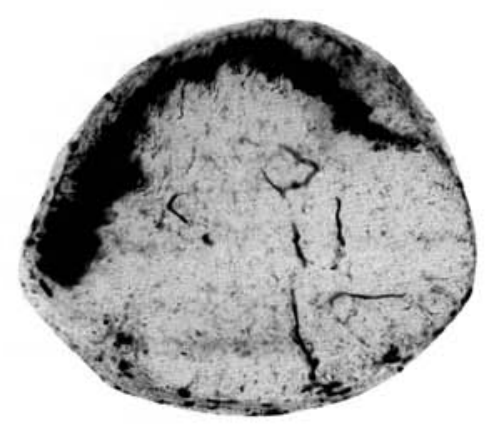

E

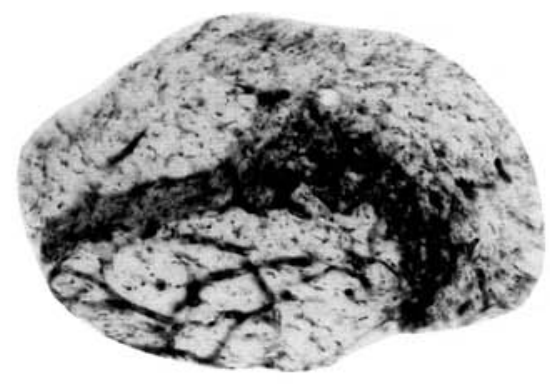

$\mathbf{F}$

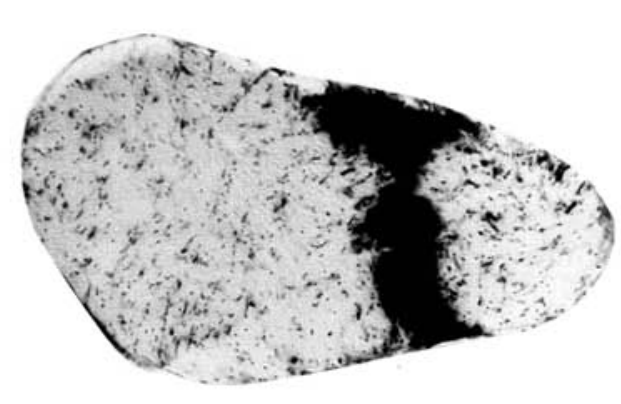

G

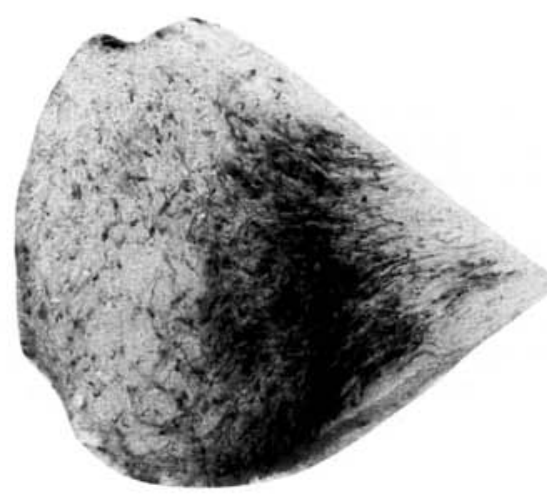

H

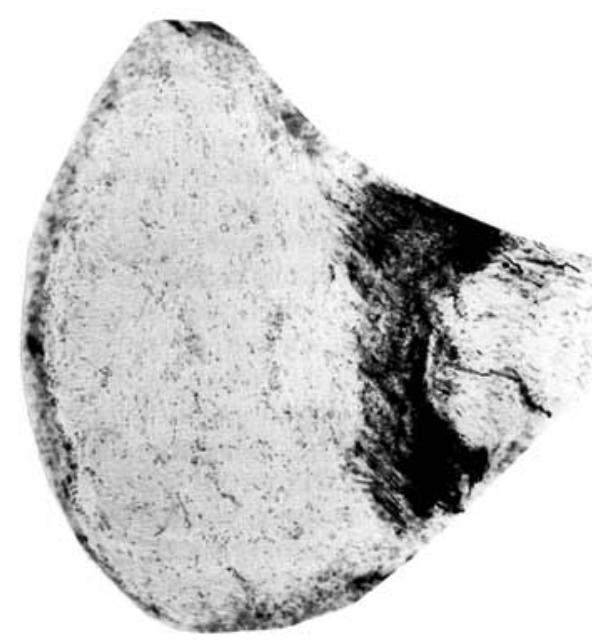



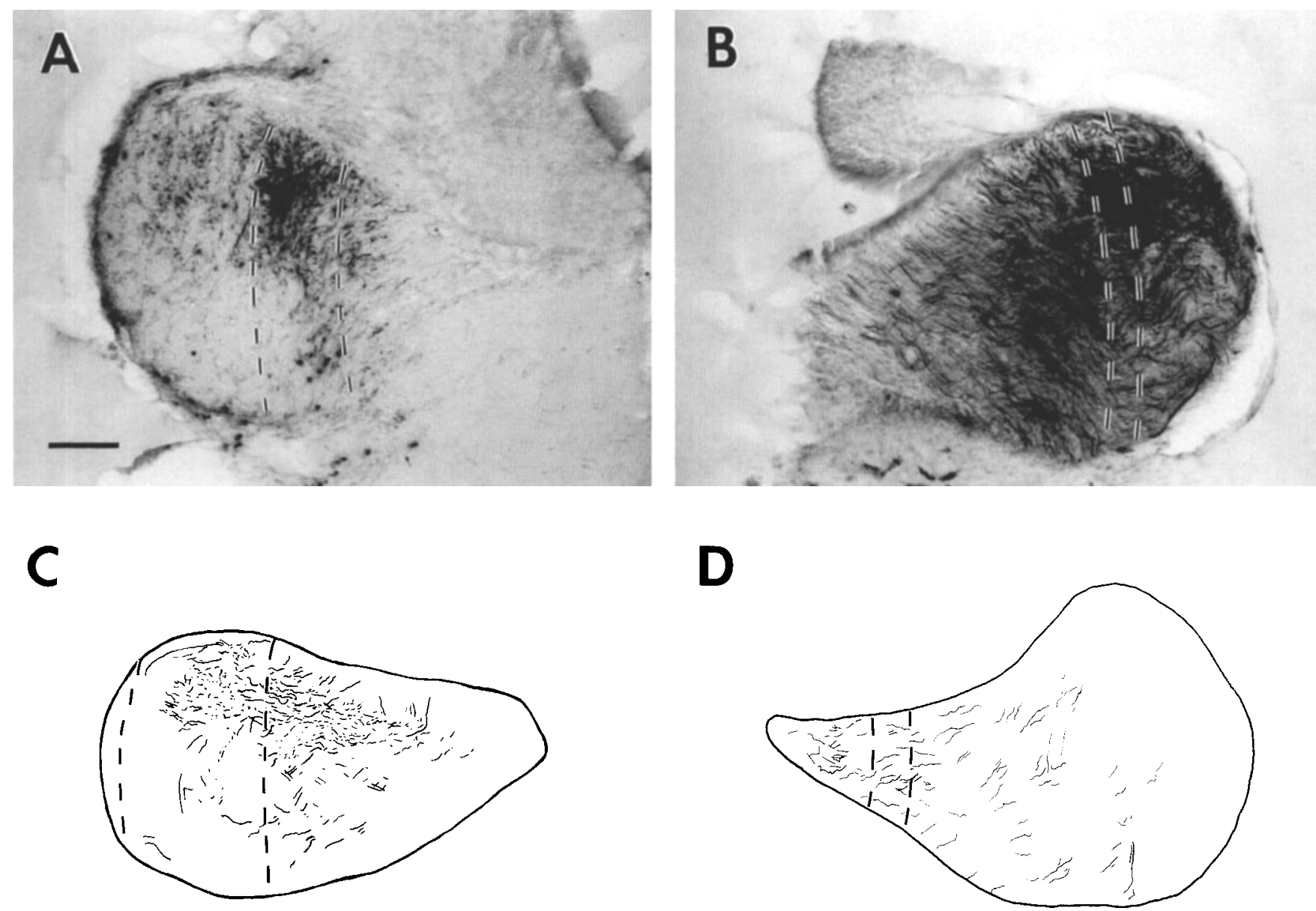

Fig. 9. The unpredictable distribution of regenerated $r$-groups at level 4. Micrographs (A,B) and camera lucida drawings (C,D) illustrating the unpre. dictable distributions of axons of common annular origin in four optic tracts. Broken lines indicate the expected location of a normal r-group in each animal inferred from the radial distance and width of the labeled retinal annuli (not shown). Medial is to the center, rostrodorsal up. Calibration bar is $100 \mu \mathrm{m}$. A: An r-group (60 days postcrush) originating from a partial

annulus at $57-70 \%$ radial distance from the optic disc in the ventral hemiretina. B: An r-group (196 days postcrush) originating at $68-75 \%$ radial distance from the optic disc in the nasal half of the retina. C: An r-group (60 days postcrush) originating from a broad annulus with two components; one at $58-75 \%$ radial distance in nasal retina joined to a second component at $90-100 \%$ radial distance in ventral retina. D: An r-group (196 days postcrush) originating at $34-40 \%$ radial distance in ventronasal retina.

Fig. 8. A comparison of regenerated (54 days postcrush) and intact $r$ groups at levels 0,3 , and 4. Camera lucida drawings of two left retinas (A,B), and micrographs $(\mathrm{C}-\mathrm{H})$ of cross sections through the correspondin pathways. Sections illustrated side by side were cut at the same level. The four panels on the left are from the experimental and the four on the right from the normal pathway. Calibration bar in $\mathrm{A}$ is $1.0 \mathrm{~mm}$ and also applies to $\mathrm{B}$. Calibration bar in $\mathrm{C}$ is $100 \mu \mathrm{m}$ and applies to $\mathrm{C}-\mathrm{H}$. A: In the experimental retina, backfilled ganglion cells occupy an almost complete annulus with a gap in the nasoventral quadrant. In addition, a few cells in central retina were also labeled. $D$, dorsal; $V$, ventral; $N$, nasal; $T$, temporal. B: In the control retina, backfilled ganglion cells occupy a complete annulus slightly more central than the one in A. Conventions as in A. C: At level 0 (nonregenerated portion) of the experimental nerve the $r$-group is clustered in a dorsal band, extending temporonasally. The label in the few axons originating from most central retina is too weak to be detected in the micrograph. D: In the normal, the r-group is similarly placed and longer than in C, reflecting the complete retinal annulus. E: At level 3 the experimental r-group extends along the long (mediolateral) axis of the cross section. Orientation in $\mathrm{E}-\mathrm{H}$ is rostrodorsal up, medial to the right. $\mathrm{F}$ : In the normal, the control r-group extends along the short axis, perpendicular to the orientation of the regenerated r-group in E. G: At level 4 the experimen tal r-group is concentrated medially and oriented roughly vertically. $\mathbf{H}$ : In the normal, the r-group is similarly concentrated medially and oriented vertically but is more compact than the regenerated $r$-group in $G$.

\section{DISCUSSION}

\section{Order along the $\mathbf{r}$-axis is severely, and perhaps completely, degraded}

We find that the r-order is not discernible in the regenerated pathway, confirming an earlier abstract (Dawnay, '81). The specific observation that r-groups are dispersed in the tectal stratum opticum of the regenerate is consistent with previous studies in which HRP was retrogradely transported from fascicles in the tectal fiber layer (Cook, '83; Stuermer and Easter, '84a). But our conclusion differs from that of Stuermer ('86); she also found abnormal fiber paths on the tectum but emphasized some reestablishment of $r$ order. We have not confirmed that result. We cannot offer an explanation for this discrepancy.

Bodick and Levinthal ('80) have suggested that the $r$ order in the normal pathway is a result of mechanical factors that guide the axons into the subpial layer and of the spatiotemporal pattern of retinal growth. Each new generation of ganglion cells is born at the retinal edge, and all the axons grow in between the pia on one side and their predecessors on the other. They remain there all the way to 

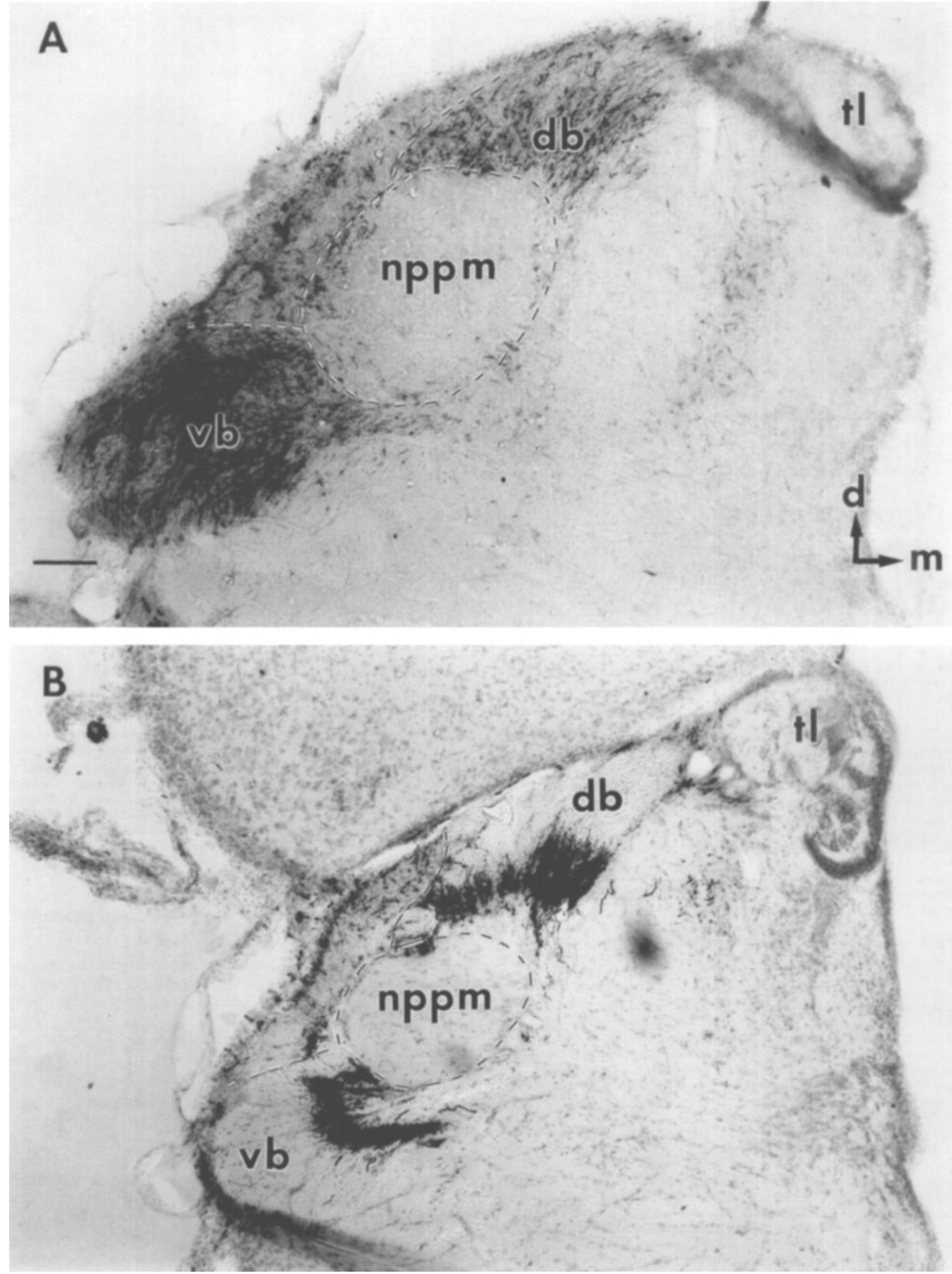

Fig. 10. A comparison of regenerated (54 days postcrush) and intact $r$ groups at level 5 . A pair of micrographs of cross sections through an experimental (A) and an intact (B) pathway. Same animals as in Figure 8 . The
contours of the brachia are partly outlined. Rostrodorsal (d) is up, medial (m) to the left. $\mathrm{db}$, dorsal brachium; vb, ventral brachium: tl, torus longitudinalis. Calibration bar is $100 \mu \mathrm{m}$. A: In the experimental brachia axons of the r-group are scattered throughout. B: In the control brachia the r-group the r-group are scattered throughout. B: In the control brachir
forms two dense bands, aligned parallel to the pial surface. 

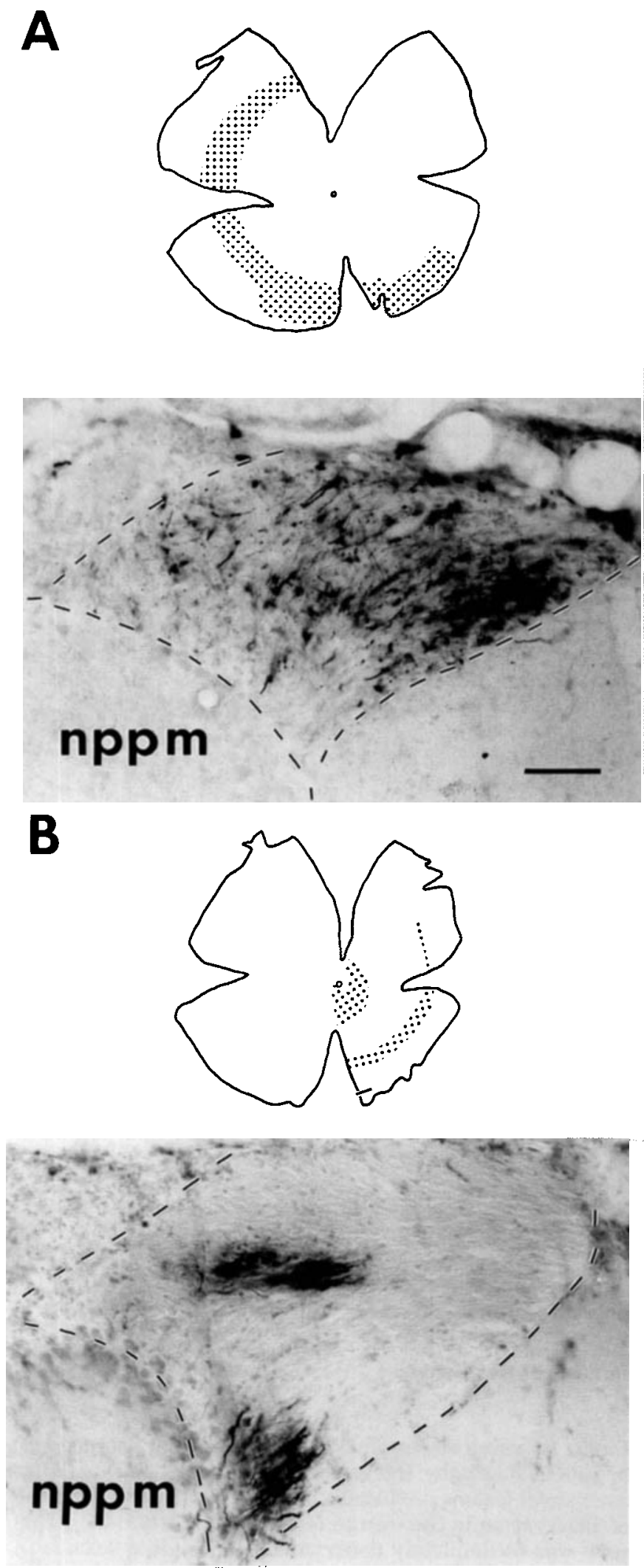

the tectum (Bernhardt and Easter, '86). As a result, over many generations, the $r$-axis is built up.

During regeneration, the conditions favoring r-order are missing. The magnitude of retinal growth is negligible during regeneration. Easter et al. ('81) estimate that 40-50 new ganglion cells are added daily in adult goldfish. Over the roughly 80 days required for complete regeneration (Meyer, '80; Murray and Edwards, '82), about 3,200-4,000 new ganglion cells would be born, a negligible number in the presence of the $100,000-200,000$ regenerating axons (Easter et al., ' 81 ). The idea that the subpial region is favorable for new optic axons is widely supported by work in fish (Scholes, '79; Easter et al., '84), amphibians (Gaze and Grant, '78), birds (Rager, ' 80 ; Krayanek and Goldberg, '81), and mammals (Silver, '84; Silver and Rutishauser, ' 84 ; Walsh and Guillery, '85; Walsh, '86). The subpial region may support axonal outgrowth because it provides a low mechanical resistance to growth or by virtue of certain chemicals found there that could provide an adhesive substrate, e.g. the neural cell adhesion molecule (N-CAM) (Silver and Rutishauser, '84) and laminin (Hopkins et al., '85). But the superficial part of the pathway is a very small part and insufficient to accommodate all the regenerating axons, which fill the entire pathway (Murray, '82). Our results indicate that the regenerating axons dispersed throughout the cross section, no longer clustered with others of their generation.

We conclude that the $r$-axis probably lacks an r-specific guidance cue. This issue is examined in greater detail in the companion paper (Bernhardt et al., '88).

\section{Order along the $\theta$-axis is reestablished}

The $\theta$-group dispersed at the lesion site, reaggregated more proximally, and formed an approximately normal $\theta$ map by abrupt shifts of position in the cross section of the pathway. In all respects, this is a normal pattern, crudely similar to what has been observed in intact pathways (Scholes, '79; Easter et al., '81; Bunt, '82; Bernhardt and Easter, '86; Fraley and Sharma, '86; Springer and Mednick, ' $86 \mathrm{~b})$.

The trivial explanation for regenerated $\theta$-order is that it originated as a consequence of the initial order on the retinal side of the lesion. According to this explanation, the regenerated axons remained ordered because of their initial "momentum." This explanation can be ruled out by the evidence for dispersion of axons at the crush site and for rearrangements in the tract and reaggregation. These suggest active, not passive, processes.

Our emphasis on the orderly aspects of the regenerated fiber topography in the brachia and the tectal fiber layer contrasts with previous reports that emphasized the aber-

Fig. 11. Another comparison of regenerated (A) and intact (B) $r$-groups at level 5 . Camera lucida tracings, indicating the retinal origins of the labeled axons are oriented such that dorsal is toward the top. Micrographs are aligned such that the pial surface is toward the top. The contours of the brachia are outlined. For orientation compare to db in Figure 10. Calibration bar is $2.0 \mathrm{~mm}$ for the retinae, $50 \mu \mathrm{m}$ for the micrographs. A: Regenerated axons ( 60 days postcrush) originating from an annulus in the left retina are scattered along the superficial to deep axis of the right dorsal brachium. The labeled axons are more concentrated away from nppm, in keeping with the predominantly nasal retinal origins of the axons. B: Normal axons originating from a central and a more peripheral annulus in this (left) control retina form two distinct bands in the right dorsal brachium. These bands are aligned parallel to the pial surface along the long axis and well separated along the superficial to deep axis of the brachium. The labeled axons are close to nppm, appropriate to their temporal sectoral origins. 

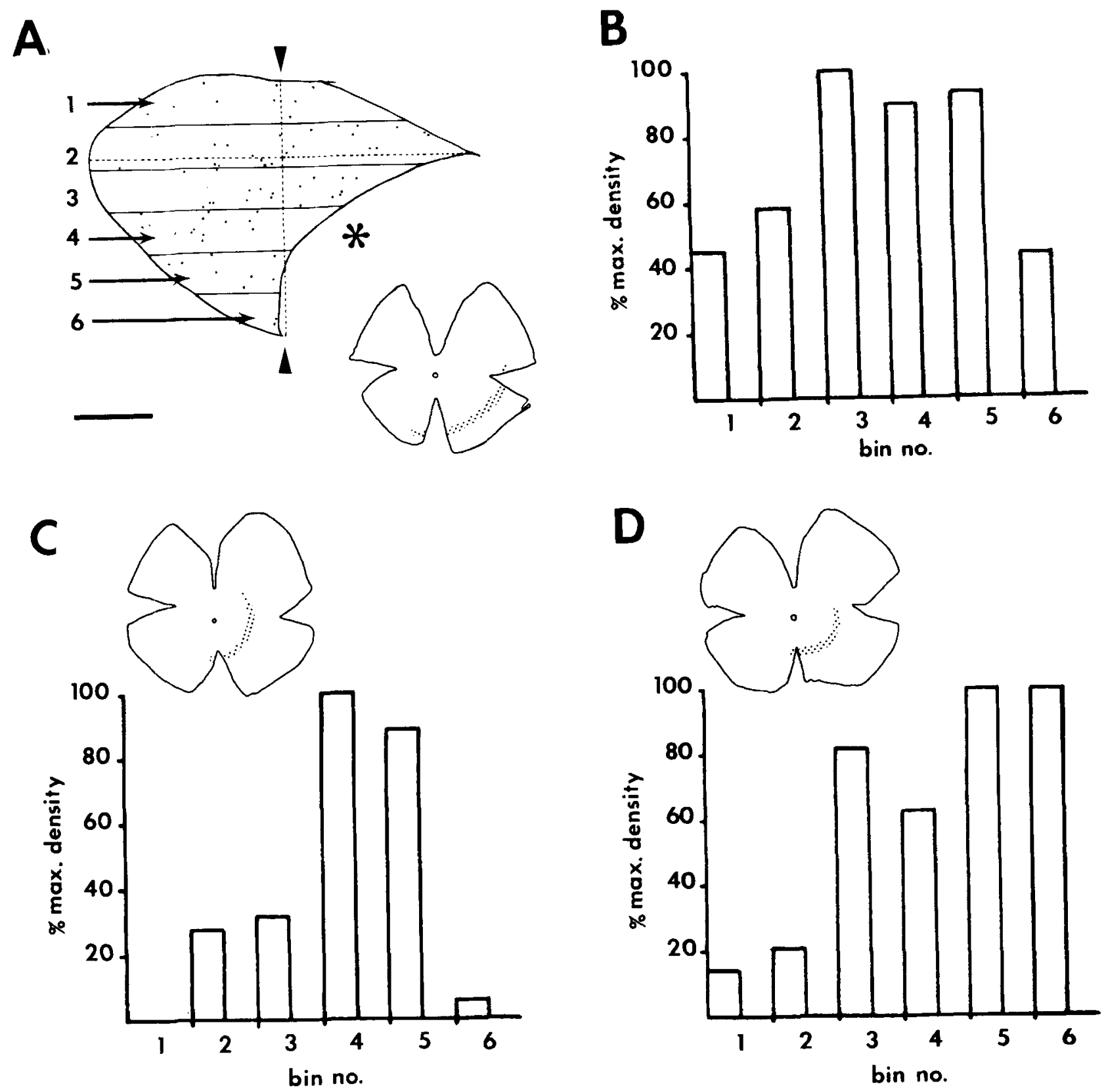

Fig. 12. Quantitative evidence against the reestablishment of $r$-order at level 5 . Compare with Figure 6. A: Axons (indicated as dots), originating from a partial retinal annulus at about $65-75 \%$ of the radial distance from the optic disc (see inset), are widely scattered throughout the cross section of an experimental left dorsal brachium. Axonal densities were determined in six bins (1-6) of equal width parallel to the long axis of the brachium (dashed line-for definition see Fig. 6). These bins extend roughly parallel (dashed line-for definition see Fig. 6). These bins extend roughly parallel The densities (axons per unit area, expressed as the percentage of the

rant trajectories of regenerated axons (Horder, '74; Meyer, '80; Cook, '83; Stuermer and Easter, '84a). The studies of Cook ('83) and Stuermer and Easter ('84a) probed only the r-order among the regenerated axons and concluded that it was degraded in regeneration. We agree. Horder ('74) and Meyer ('80) examined the regenerated axonal topography by lesioning the brachium or the optic fiber layer, respec-

maximum density in the brachium) within each bin. Inset in A gives the retinal origins. These axons are normally expected to form a sharp peak in bin 2 or 3 (compare Fig. 11B, superficial $r$-band). In the regenerate 65 days postcrush) they form a broad central peak similar to axons of different radial origins shown in Figure 12C,D. C,D: Bar graphs plotting the normalized densities of axons ( 65 and 196 days postcrush, respectively) from two ther experimental r-groups (see insets), both originating from partial retinal annuli at about $30-40 \%$ of the radial distance from the optic disc. They are distributed very differently in the regenerated brachia.

tively. By using electrophysiological recording (Horder, '74) or autoradiography (Meyer, '80), they demonstrated that such small lesions produced a predictable and compact zone of denervation in the tectum of otherwise normal fish. This zone was incompletely denervated after similar lesions in the regenerated pathway, indicating that at least sorne axons had taken abnormal routes to their tectal targets. 


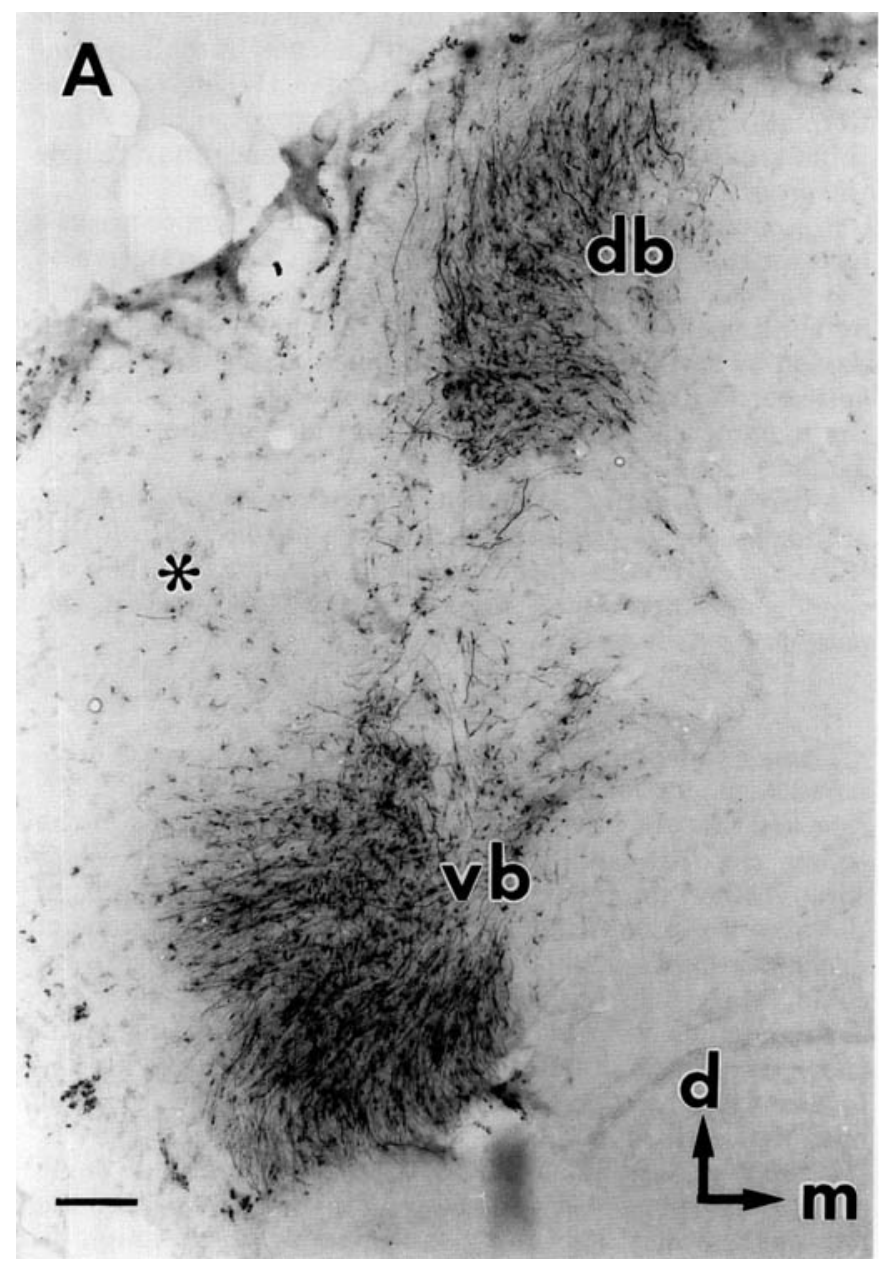

Fig. 13. Dissimilar tectal innervation patterns of regenerated and intact r-groups. A pair of micrographs of cross sections through the rostral pole of the tectum, illustrating the innervation pattern established by a regenerated (54 days post crush) r-group (A) and an intact one (B). Same animal as in Figures 8 and 10. Rostrodorsal (d) is up, medial (m) to the right. db, dorsal

The aberrant regenerates were emphasized because they had not previously been described and because the methods were designed to detect them rather than the regenerates that had followed the normal trajectories. In contrast, our method labeled most of the axons from a given sector and revealed that many of them occupied positions in the pathway appropriate to their sectoral origins. Thus, our method was similar to that of Attardi and Sperry ('63) and, like those authors, we emphasize the pattern typical of the majority of axons. As might be expected, given an interval of 24 years, our method had certain advantages over theirs. We labeled a more restricted set of axons than they did, and our evaluation was more accurate than theirs, as we had a more detailed description of the normal pathway against which to compare the regenerates.

In remarking that a particular method biases one to emphasize either the order or the disorder, we are reminded of the recent controversy over the retinogeniculate pathway of the cat. Horton et al. ('79) labeled a few axons of neigh. boring ganglion cells, followed them through the pathway, and emphasized their dispersion. Guillery and his collaborators (Torrealba et al., '81, '82) labeled much larger num-

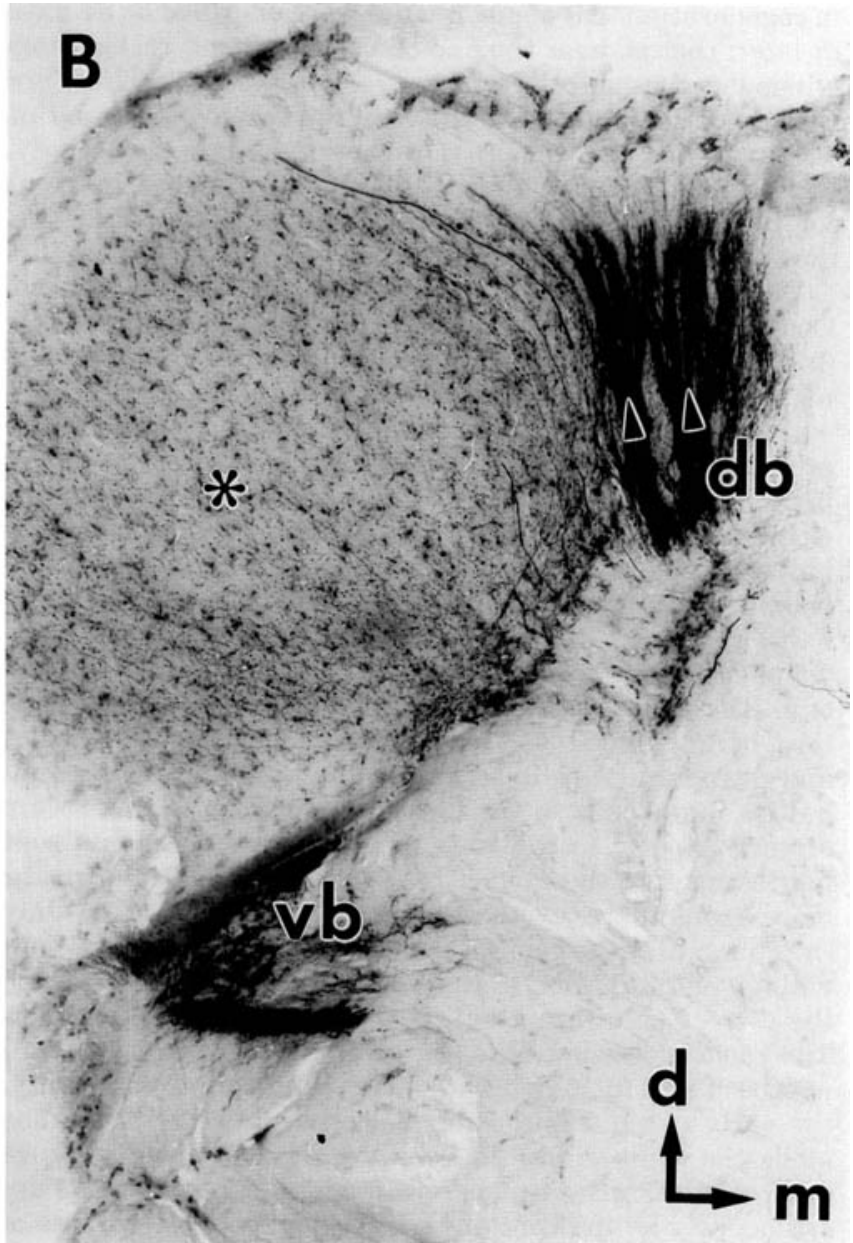

brachium; vb, ventral brachium; asterisks mark tectum. Calibration bar is $100 \mu \mathrm{m}$. A: Both brachia have joined the tectum. Labeled axons are evenly spread out both in the brachia and on the tectum. B: The dorsal brachium has fused with the tectum, and its labeled axons enter the tectum in bundles (arrowheads). The ventral brachium has not yet fused with the tectum.

bers and emphasized their coherence, while acknowledging the ectopic axons. Our approach resembles theirs.

\section{Source of the $\theta$-order}

The reestablishment of order along the $\theta$-axis may have occurred during or after regeneration. If the regenerating axons were able to navigate by following the same $\theta$-specific cues that ordered the original projection, and if those same cues remained available, then it would follow that the normal order would be reestablished. Alternatively, it could be argued that regenerative outgrowth may have been random, uninfluenced by any cues, and that the order resulted from a later selective disappearance of axons from the "incorrect" regions.

The report by Becker and Cook ('87) argues in favor of the second alternative, postregeneration sharpening. They labeled the dorsal brachium with HRP and found substantially more retrogradely labeled cells in the wrong (dorsal) hemiretina early in regeneration (24-28 days) than late (after 42-70 days). Hartlieb and Stuermer ('87), while noting early brachial selection, also described secondary changes in the fiber topography during the second month 
of regeneration. All of our results were obtained at 54 days or later; that is, near the end of, or subsequent to, the time window proposed for secondary rearrangements. Therefore they do not help to decide between the two hypotheses. But we favor the idea of early, rather than late, selectivity, because Attardi and Sperry ('63) noted that the brachial selection was largely correct from the earliest times that they could identify regenerates.

Moreover, the retrograde method used by Becker and Cook ('87) can be misleading, as the following example (inspired by Thompson, '83) should indicate. We know that regenerating optic axons branch at the site of the lesion and perhaps beyond (Murray, '82). Suppose that each axon produces five branches, four of which select the correct brachium, and one of which selects the incorrect one. If either brachium is labeled with HRP, all the cells in both halves of the retina will be labeled; that is, the ratio, (labeled cells in the wrong half/labeled cells in the correct half), will be $1 / 1$ at early stages, a misleading result, since $4 / 5$ of the axons selected correctly. Then, suppose that half of all the axons disappear, irrespective of which brachium they occupy. One-half of the errant axons will disappear, and retrograde labeling through the brachium will reveal half as many cells in the wrong hemiretina as there were at early stages. In contrast, most of the somata that sent four axons into the correct brachium will still have one or more axons there, even after half of them disappear. Only $(1 / 2)^{4}$ or $1 / 16$ of the somata that had four axons there before will have lost all four, and so the number of labeled cells in the correct hemiretina will be $15 / 16$ the original number. The ratio of labeled cells in wrong/right hemiretinas will now be $(1 / 2) /(15 / 16)=16 / 30$, which is much less than unity, the early value. Thus, early selectivity, (4/5 of the axons made the right choice) followed by random disappearance (half of all axons disappeared) leads to a result that may erroneously be interpreted as evidence for selective loss of errant axons.

In a series of preliminary experiments we have investigated brachial selection by regenerating axons at 20-30 days after nerve crush. Using a fluorescent tracer of the carbocyanine family (Honig and Hume, '86) to circumvent the problem of unreliable transport of HRP in regenerating axons (Murray and Edwards, '82; Becker and Cook, '87) we find that axons tend to choose the brachium appropriate to their ventral or dorsal hemiretinal origins even during the initial stages of regeneration (Bernhardt, in preparation). These findings are consistent with a recently published study of the initial fiber order in the regenerated pathway of goldfish (Hartlieb and Stuermer, '87). We do not claim that the demonstrations of early selectivity (Attardi and Sperry, '63; Hartlieb and Stuermer, '87; Bernhardt, in preparation) exclude the possibility of a later pruning phase, as Becker and Cook ('87) and Hartlieb and Stuermer ('87) have described. But given the argument of the preceding paragraph, we believe that the selectivity of the later pruning may have been overemphasized. We conclude that the present evidence for sharpening of the regenerated fiber topography during the second month of regeneration does not argue against pathfinding by regenerating axons.

The crudeness of the order, relative to the normal, could be attributed to several factors. The first is the absence of a preexisting axonal substrate on which to grow. Most of the axons in the normal pathway grew in along their immediate predecessors, axons with the same $\theta$ and slightly smaller $r$. If there were $\theta$-specific interactions between growth cones and axons, as Bonhoeffer and Huf ('85) have shown in chick retina, they might synergize with the cues in the environment and contribute to the normal order. During regeneration, all axons, at least initially, must grow without other intact axons as a substrate, and this absence may reduce the order.

A second possibility is a reduced amount of guidance cue (presumably chemical) per regenerating axon, relative to the normal. If there were a fixed amount, normally shared by the trickle of new axons, and this amount had to be shared by the suddenly larger number of regenerating axons, the result could be as we have observed, particularly if the hypothetical substance were degraded by the growing axons.

A third possibility, that guidance cues are only available at the pial border, would seem to be excluded by our results. If the $\theta$-specific cues were only found superficially, then we would not expect to see $\theta$-order reestablished deep in the pathway-but it was.

\section{CONCLUSIONS}

These results refine our description of the process of optic nerve regeneration. The earliest papers (e.g., Matthey, '27) reported that the regenerated axons grew chaotically at the lesion site but resumed generally parallel trajectories through the remainder of the pathway into the tectum. In the absence of methods that could label subpopulations of axons, the possibility of topographic order in the pathway could not be investigated. Attardi and Sperry ('63) provided such a method and concluded that most regenerating axons chose the correct brachium and grew more or less directly toward the correct termination site. Others confirmed that most regenerates made the correct brachial choice (Horder, '74; Stuermer and Easter, '84a; this paper), but these same authors described aberrant trajectories in the tract and the tectum. The most strikingly so were reported in amphibians by Fujisawa and his collaborators (Fujisawa, '81; Fujisawa et al., '82) and confirmed in fish (Schmidt et al., '87). The existence of enormous terminal arbors at early stages of regeneration, and the apparently chaotic trajectories at late stages, led to the proposition that termination in the tectum was a trial and error phenomenon. The regenerating axon sent out growth cones in a variety of directions, perhaps even at random, maintained the one or ones that found an appropriate site, and withdrew those at inappropriate locations.

Our results suggest that the process is more ordered, as more regenerating axons sought the proper locations, at least along the $\theta$-axis, in the tract, brachium, and tectal stratum opticum. This shift in emphasis-from disorder to order-is attributable to the fact that our method labeled substantial numbers of axons and to the detailed description of the normal pathway that has recently become available.

But we consistently found no order regenerated along the $r$-axis. How does it normally arise and to what extent is it a result of growth cones tracking the axons from nearby retinal regions? These questions are asked in the next paper (Bernhardt et al., '88).

\section{ACKNOWLEDGMENTS}

This work was supported by research grant EY-00168 to S.S.E. and by a grant from the Janggen-Poehn Stiftung to R.B. 
We thank Ms. Celeste Malinoski for technical assistance, Mr. David Bay for photographic assistance, and Ms. Linda Freeman for typing the paper.

\section{LITERATURE CITED}

Adams, J.C. (1981) Heavy metal intensification of DAB-based HRP reaction product. J. Histochem. Cytochem. 26:775.

Attardi, D.G., and R.W. Sperry (1963) Preferential selection of central pathways by regenerating optic fibers. Exp. Neurol. 7:46-64.

Becker, D.L., and J.E. Cook (1987) Initial disorder and secondary retinotopic refinement of the regenerating axons in the optic tract of goldfish: Signs of a new role for collateral loss. Development 101:323-337.

Bernhardt, R., and S.S. Easter (1986) Map of retinal position onto the cross section of the optic pathway in goldfish. J. Comp. Neurol. 254:493-510.

Bernhardt, R., and S.S. Easter (1987) Regenerating axons reestablish normal sectoral order in the optic brachia of goldfish. Neurosci. Abstr. 13:256

Bernhardt, R., S.S. Easter, Jr., and P.A. Raymond (1988) Axons added to the regenerated visual pathway of goldfish establish a normal fiber topography along the age-axis. J. Comp. Neurol. 277:420-429.

Bernhardt, R., S.S. Easter, P.A. Raymond, and P. Rivlin (1986) Axons added to the optic pathway subsequent to its regeneration reestablish the normal fiber topography lost during regeneration. Neurosci. Abstr. 12:1211.

Bodick, N., and C. Levinthal (1980) Growing optic nerve fibers follow neighbors during embryogenesis. Proc. Natl. Acad. Sci. USA 77:4374-4378.

Bonhoeffer, $R$, and J. Huf (1985) Position dependent properties of retinal axons and their growth cones. Nature 315:409-410.

Bunt, S.M. (1982) Retinotopic and temporal organization of the optic nerve and tracts in the adult goldfish. J. Comp. Neurol. 206:209-226.

Cook,J.E.(1983) Tectal paths of regenerated optic axons in the goldfish Evidence from retrograde labeling with horseradish peroxidase. Exp. Brain Res. 51:433-442.

Dawnay, N.A.H. (1981) Fiber ordering within regenerated optic pathways of goldfish. J. Physiol. (Lond.) 317:76P-77P.

Easter, S.S., and R. Bernhardt (1987) Axonal pathfinding in the retinotectal projection of goldfish. J. Anat. 152:248.

Easter, S.S., B. Bratton, and S.S. Scherer (1984) Growth related order of the retinal fiber layer in goldfish. J. Neurosci. 4:2173-2190.

Easter, S.S., A.C. Rusoff, and P.E. Kish (1981) The growth and organization of the optic nerve and tract in juvenile and adult goldfish. J. Neurosci. 1:793-811.

Fraley, S.M., and S.C. Sharma (1986) Retinal topography in the optic tract of adult goldfish. Neuroscience 19:1363-1380.

Fujisawa, H. (1981) Retinotopic analysis of fiber pathways in the regener ating retinotectal system of the adult newt Cynops pyrrhogaster. Brain Res. 206:27-37.

Fujisawa, H, N. Tani, K. Watanabe, and Y. Ibata (1982) Branching of regenerating retinal axons and preferential selection of appropriate branches for specific neuronal connection in the newt. Dev. Biol. 90:4357.

Gaze, R.M., and P. Grant (1978) The diencephalic course of regenerating retinotectal fibers in Xenopus tadpoles. J. Embryol. Exp. Morphol. 44:201-216.

Hanker, J.S., P.E. Yates, C.B. Metz, and A. Rustoni (1977) A new specific sensitive and non-carcinogenic reagent for the demonstration of horseradish peroxidase. Histochem. J. 9:789-792.

Hartlieb, E., and C.A.O. Stuermer (1987) Preferential loss of collaterals from goldfish retinal axons in the optic tract is delayed by tetrodotoxin. Neurosci. Lett. 79:1-5.

Hopkins, J.M., T.S. Ford-Holevinski, J.P. McCoy, and B.W. Agranoff (1985)
Laminin and optic nerve regeneration in the goldfish. J. Neurosci. 5:3030-3038.

Horder, T.J. (1974) Changes of fiber pathways in the goldfish optic tract following regeneration. Brain Res. 72:41-52.

Horton, J.C., M.M. Greenwood, and D.H. Hubel (1979) Non-retinotopic arrangement of fibers in the cat optic nerve. Nature 282:720-722.

Honig, M.G.,and R.I. Hume (1986) Fluorescent carbocyanine dyes allow living neurons of identified origin to be studied in long-term cultures. J. Cell Biol. 103:171-187.

Krayanek, S., and S. Goldberg (1981) Oriented extracellular channels and axonal guidance in the embryonic chick retina. Dev. Biol. 84:41-50.

Matthey, R. (1927) La greffe de l'oeil. Étude experimentale de la greffe de l'oeil chez le triton (Triton cristatus). Roux Arch. 109:326-341.

Meyer, R.L. (1980) Mapping of the normal and regenerating retinotectal projection of goldfish with autoradiographic methods. J. Comp. Neurol. 189:273-289.

Murray, M. (1982) A quantitative study of regenerative sprouting by optic axons in the goldfish. J. Comp. Neurol. 209:352-362.

Murray, M., and M.A. Edwards (1982) A quantitative study of the reinnervation of the goldfish optic tectum following optic nerve crush. J. Comp. Neurol. 209:363-373.

Rager, G.H. (1980) Development of the retinotectal projection in the chicken. Adv. Anat. Embryol. Cell Biol. 63:1-92.

Rusoff, A.C., and S.S. Easter (1980) Order in the optic nerve in goldfish. Science 208:311-312.

Schmidt, J.T., J.C. Turcotte, M. Buzzard, and D.G. Tieman (1988) Staining of regenerated optic arbors in goldfish tectum: Progressive changes in immature arbors and a comparison of mature regenerated arbors with normal arbors. J. Comp. Neurol. 269:565-591.

Scholes, J.H. (1979) Nerve fibre topography in the retinal projection to the tectum. Nature 278:620-624.

Silver, J. (1984) Studies of factors that govern directionality of axonal growth in the embryonic optic nerve and at the chiasm of mice. J. Comp. Neurol. 223:238-251.

Silver, J., and U. Rutishauser (1984) Guidance of optic axons in vivo by a preformed adhesive pathway on neuroepithelial endfeet. Dev. Biol. 106:485-499.

Springer, A.D., and A.S. Mednick (1986a) Retinotopic and chronotopic organization of goldfish retinal ganglion cell axons throughout the optic nerve. J. Comp. Neurol. 247:221-232.

Springer, A.D., and A.S. Mednick (1986b) Simple and complex retinal ganglion cell axonal rearrangements at the optic chiasm. J. Comp. Neurol. 247:233-245.

Stuermer, C.A.O. (1986) Pathways of regenerated retinotectal axons in goldfish. 1. Optic nerve, tract and tectal fascicle layer. J. Embryol. Exp. Morphol. 93:1-28.

Stuermer, C.A.O., and S.S. Easter (1984a) A comparison of the normal and regenerated retinotectal pathways of goldfish. J. Comp. Neurol. 223:5776 .

Stuermer, C.A.O., and S.S. Easter (1984b) Rules of order in the retinotectal fascicles of goldfish. J. Neurosci, 4:1045-1051.

Torrealba, F., R.W. Guillery, E.H. Polley, and C.A. Mason (1981) A demonstration of several independent, partially overlapping retinotopic maps in the optic tract of the cat. Brain Res. 219:428-432.

Torrealba, F., R.W. Guillery, U. Eysel, E.H. Polley, and C.A. Mason (1982) Studies on retinal representations within the cat's optic tract. J. Comp. Neurol. 211:377-396.

Thompson, W.J. (1983) Lack of segmental selectivity in elimination of synapses from soleus muscle of new-born rats. J. Physiol. (Lond.) 335:343352

Walsh, C. (1986) Age-related fiber order in the ferret's optic nerve and optic chiasm. J. Neurosci. 6:1635-1642.

Walsh, C., and R.W. Guillery (1985) Age-related fiber order in the optic tract of the ferret. J. Neurosci. 5:3061-3069. 